University of Louisville

ThinkIR: The University of Louisville's Institutional Repository

8-2011

\title{
Characterization of hematite nanowire arrays synthesized by atmospheric plasma.
}

Harry Benjamin Russell

University of Louisville

Follow this and additional works at: https://ir.library.louisville.edu/etd

\section{Recommended Citation}

Russell, Harry Benjamin, "Characterization of hematite nanowire arrays synthesized by atmospheric plasma." (2011). Electronic Theses and Dissertations. Paper 1242.

https://doi.org/10.18297/etd/1242

This Master's Thesis is brought to you for free and open access by ThinkIR: The University of Louisville's Institutional Repository. It has been accepted for inclusion in Electronic Theses and Dissertations by an authorized administrator of ThinkIR: The University of Louisville's Institutional Repository. This title appears here courtesy of the author, who has retained all other copyrights. For more information, please contact thinkir@louisville.edu. 
Characterization of Hematite Nanowire Arrays synthesized by Atmospheric Plasma

\author{
By
}

Harry Benjamin Russell

B.S., University of Louisville, 2009

\author{
A Thesis \\ Submitted to the Faculty of the \\ University of Louisville \\ J.B. Speed School of Engineering \\ as Partial Fulfillment of the Requirements \\ For the Professional Degree \\ MASTER OF ENGINEERING \\ Department of Chemical Engineering
}

August 2011 
Characterization of Hematite Nanowire Arrays synthesized by Atmospheric Plasma Submitted

by:

Harry Benjamin Russell

A Thesis Approved on

(Date)

By the Following Reading and Examination Committee:

Mahendra K. Sunkara, PhD, Thesis Director

Moises A. Carreon, $\mathrm{PhD}$

Gamini Sumanasekera, PhD 


\section{ACKNOWLEDGEMENTS}

The author would first and foremost like to thank Mahendra K. Sunkara for his great help, advice and motivation. Secondly, thanks from the author go to Dr. Moises A. Carreon and Gamini Sumanasekera for serving on the author's MENG committee and offering advice along the way. Thanks also go to Boris Chernomordik for blazing the trail on hematite nanowire research at UofL and allowing a continuation of his research after his departure. Thanks also go to US Department of Energy, KY NASA EPSCOR, KY Space Grant Consortium, the Conn Center for Renewable Energy and Zeon Specialty Chemicals for financial assistance. 


\begin{abstract}
Hematite synthesized by low pressure oxygen plasma has been shown previously to exhibit promising characteristics as a photo-anode for a photoelectrochemical water splitting cell. It is cheap, stable and has a $2.1 \mathrm{eV}$ bandgap and exhibits a significant photoactivity due to the presence of a thin interfacial layer and oxygen vacancy planes throughout the sample. Unfortunately, due to the nature of vacuum systems, this process would be difficult to scale-up and as such atmospheric plasma synthesis was explored.
\end{abstract}

Photoactive hematite nanowire arrays were synthesized using an atmospheric microwave plasma jet reactor. They exhibited all of the characteristics of those synthesized under low pressure except for poor photoactivity due to a thin amorphous oxide layer on the surface layer. This oxide layer was removed by hydrofluoric acid etching and subsequently produced a significant photoactivity. Further synthesis improvements have yielded hematite nanowire arrays with no amorphous oxide layer present. Removal of nanowires from the nanowire array decreased the subsequent photoactivity of the electrode, indicating that photoactivity is due to the nanowires present on the sample and not due to the thin interfacial layer. Testing at different light intensities under constant voltage shows that photoactivity of hematite nanowire arrays is linearly dependent on light intensity. 


\section{TABLE OF CONTENTS}

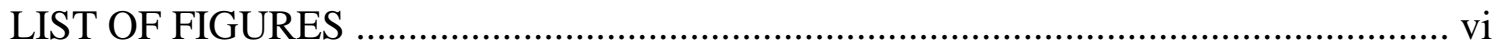

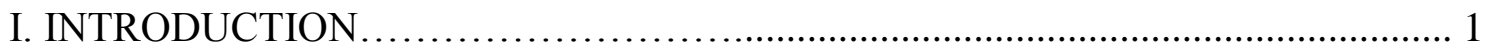

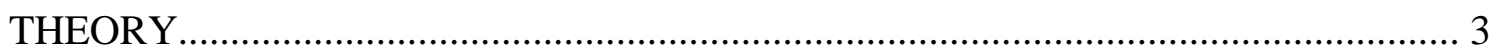

Photoelectrolysis of Water...................................................................................... 3

Principles of Semiconductors: ................................................................................ 7

Requirements for a viable PEC material............................................................... 17

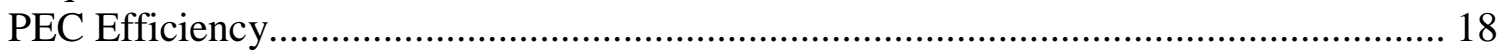

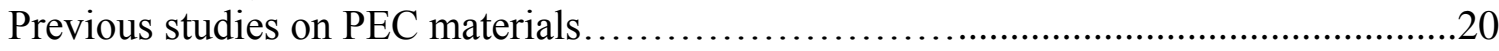

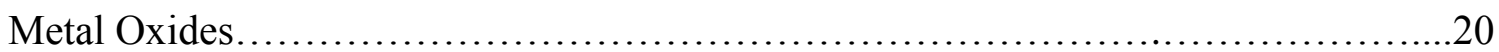

Elements from group IV and III-V elemental combinations.........................23

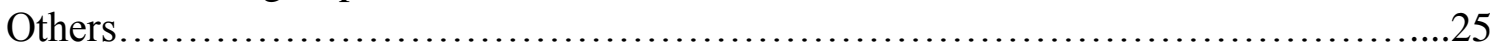

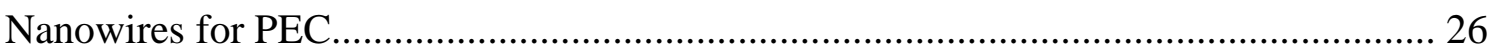

Purpose and Intent of this Study.......................................................................... 29

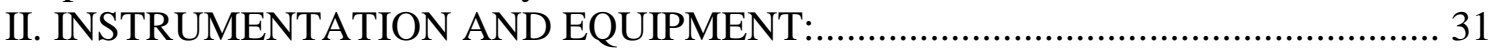

Atmospheric Microwave Plasma Jet Reactor …………............................................ 31

Photoelectrochemistry Setup ……………………............................................ 32

Scanning Electron Microscope ……………………............................................. 34

Transmission Electron Microscope ............................................................................... 34

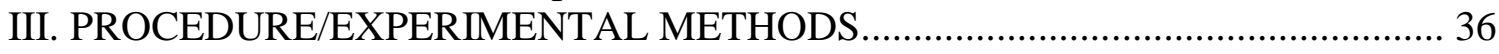

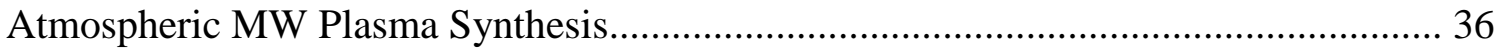

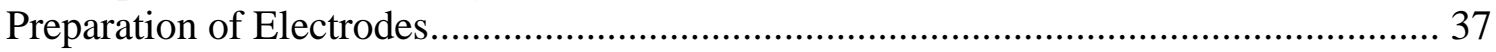

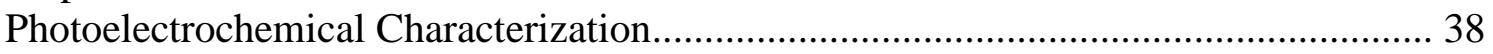

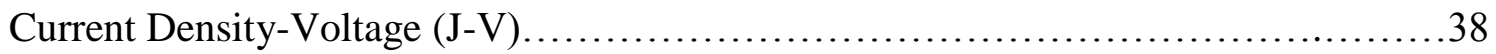

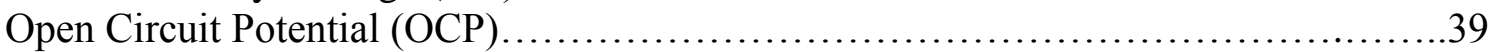

IV. RESULTS AND DISCUSSION................................................................ 40

Synthesis and Characterization of HP Plasma Oxidized Hematite NW Arrays.............. 40

HP Plasma versus LP Plasma Synthesis......................................................................... 44

Confirmation of Photoactivity due to Nanowires....................................49

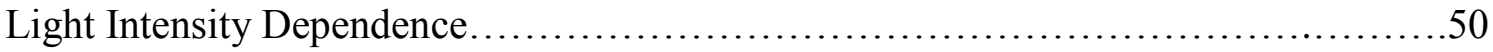

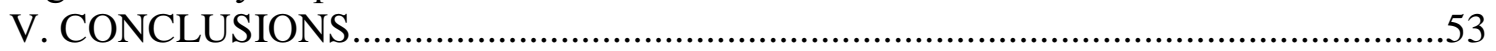

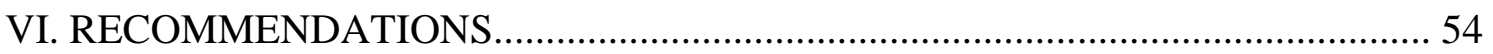

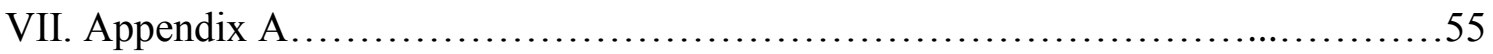

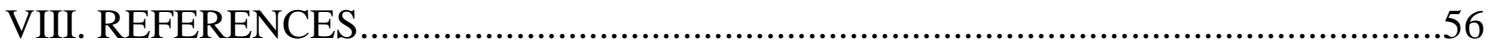

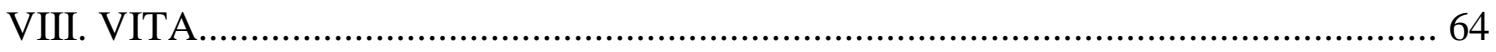




\section{LIST OF FIGURES}

FIGURE 1 - Standard AM0 and AM 1.5 solar spectra.............................

FIGURE 2 - Basic three-electrode photoelectrochemical cell....................... 7

FIGURE 3 - Electron Hole Pair creation over a band gap............................

FIGURE 4 - Direct and Indirect Band Diagrams.............................. 10

FIGURE 5 - Band schematic for $\mathrm{p}-\mathrm{n}$ junction.............................. 12

FIGURE 6 - Possible Band diagrams for water-splitting scenarios ................. 16

FIGURE 7 - Thermal vs. Plasma summary..................................29

FIGURE 8 - Microwave Plasma Jet Reactor Schematic............................ 32

FIGURE 9 - Schematic of Three Electrode Set-up used...............................

FIGURE 10 - Photograph of assembled electrode and parts...................... 37

FIGURE 11 - SEM of HP Plasma Hematite NW Array...........................40

FIGURE 12 - Raman Spectra for HP Plasma NW Array........................41

FIGURE 13 - UV-Vis for HP Plasma Hematite NW Array.......................43

FIGURE 14 - OCP measurement of Hematite NW Array.........................44

FIGURE 15 - HRTEM of several hematite NWs.............................45

FIGURE $16-\mathrm{J}-\mathrm{V}$ comparison before and after HF Etchin........................46

FIGURE 17 - Reproducibly photoactive HP Plasma samples......................48

FIGURE 18 - Effect of NW Removal......................................50

FIGURE 19 - Light Intensity Dependence....................................52

FIGURE 20 - HRTEM of hematite NW ...................................53 


\section{INTRODUCTION}

Society as we know it today depends largely on its use of energy. Sources of energy are either becoming scarcer or less feasible every day. The energy demand for the world was 15+ TW in 2008 and with less industrialized countries such as India rapidly growing; the energy demand is expected to double by 2050 . With a single nuclear power plant producing approximately 1TW of energy, over 15000 nuclear power plants would currently be needed to power the earth, and even if uranium sources on earth were not limited, the vast amount of nuclear waste that would be created would be a deterrent on its own. Also, after the recent natural disaster in Japan, the potential dangers of nuclear reactors have been exposed and the political climate for introducing more nuclear reactors is definitely unfavorable. Fossil fuels such as coal, natural gas and oil contribute the vast majority of the global energy demand. The supply of fossil fuels is limited, their use contributes to environmental problems, and their control causes conflicts between states. When these resources near their end, energy costs will shoot through the roof, the economy will slow causing social unrest. For the human race to progress, energy needs to be safe for the environment, affordable and available to the masses around the globe.

Renewable Energies offer an alternative to conventionally used energy. Those that are most promising include biomass, geothermal, hydro, hydrogen, solar, wave and wind. Hydro, wave, geothermal and to some extent wind are dependant upon location and so can only provide a worthwhile amount of energy to areas in the vicinity. At this time, biomass puts a strain on global agricultural means, potentially produces just as 
many adverse emissions and may take more energy put into processing the biomass than is gained by its use. With over 120,000 TW of solar energy falling incident to the earth's surface, solar energy by itself could solve the global energy crisis. But even if a way were found to harness all of this energy, it is only usable during daylight hours and this can obviously be obstructed on a cloudy day as well. Thus this energy must be stored in some way such as a battery or by pumping water up a mountain to take advantage of the hydro energy provided at night. Both of these options are expensive and still have a long way to go until they are feasible on a mass scale.

The most renewable way to store this energy would be to take advantage of the water-splitting reaction, which produces hydrogen that could be stored and used later, as a fuel to warm homes or combined in a fuel cell with oxygen to produce electricity. One way to carry this reaction forward would be by utilizing an electrolyzer powered by solar panels. While this is a very plausible scenario for hydrogen production, electrolyzers require the use of platinum as a catalyst, which is now feasible on a global scale as it is a precious metal. Also, energy is lost due to this being a 2 step process going from the solar panels to the electrolyzer. The alternative is to utilize a semiconductor $\mathrm{p} / \mathrm{n}$ junction device that can take in solar radiation and electrochemically split water into oxygen and hydrogen at the surface of the anode and cathode respectively. 


\section{THEORY}

\section{Photoelectrolysis of Water}

The water splitting reaction or electrolysis of water was first performed by use of $\mathrm{TiO}_{2}$ catalysis by Fujishima and Honda in $1972^{1}$. The reaction carries out as the follows:

$$
2 \mathrm{H}_{2} \mathrm{O}(l) \leftrightarrow 2 \mathrm{H}_{2}(g)+\mathrm{O}_{2}(g) \quad \Delta E^{\circ}=-1.23 \mathrm{eV}
$$

The water splitting reaction is the net reaction of two partial reactions that occur at the two separate electrodes. At the cathode, gaseous hydrogen is produced by a reduction reaction:

$$
2 H^{+}(a q)+2 e^{-} \leftrightarrow H_{2}(g) \quad E^{\circ}=0.00 \mathrm{eV}
$$

The potential at which this reaction occurs is defined in electrochemistry as the Normal Hydrogen Electrode (NHE). Gaseous oxygen evolution occurs at the anode by the following oxidation reaction.

$$
2 \mathrm{H}_{2} \mathrm{O}(l) \leftrightarrow \mathrm{O}_{2}(g)+4 H^{+}(a q)+4 e^{-} \quad E^{\circ}=-1.23 \mathrm{eV}
$$

Because the half-reactions occur at the two separate electrodes, hydrogen and oxygen can be produced separately allowing them to be stored for later use. 
The water splitting reaction does not happen spontaneously, and requires a minimum of $1.23 \mathrm{eV}$ to carry forth the reaction as shown in equation 1. Correlating photon energy input to the photon wavelength, photon wavelength required to provide $1.23 \mathrm{eV}$ to drive this reaction can be obtained:

$$
\lambda=\mathrm{h} c / e E, \mathrm{c}=2.998 * 10^{17} \frac{\mathrm{nm}}{\mathrm{s}}, h=6.625 * 10^{-34}, e=1.60 * 10^{-19} \mathrm{C}
$$

(where $\mathrm{c}$ is the speed of light under vacuum, $\lambda$ is the photon wavelength in nanometers, $\mathrm{e}$ is the electronic charge magnitude, $\mathrm{h}$ is Planck's constant and $\mathrm{E}$ is the energy of the photon in $\mathrm{eV}$ ). Using this equation, a wavelength of $1010 \mathrm{~nm}$ or less will provide photons with $1.23 \mathrm{eV}$ of energy or more in order to drive the water splitting reaction. Sections of the solar spectrum are filtered out by the earth's atmosphere and are represented in the integrated spectral solar irradiance. Also, the intensity of radiation decreases with distance as expected from the Beer-Lambert Law. The Air Mass standard was formulated to account for the Beer-Lambert law as well as obstructions caused by the earth's atmosphere. The Air Mass standard is referred to by "AM-X" where " $\mathrm{X}$ " is the air mass coefficient:

$$
X=\frac{I}{I_{0}}=\frac{I_{0}}{I_{0} \cos \theta}=\frac{1}{\cos \theta}
$$

with $\mathrm{I} 0$ is the light intensity prior to reaching the atmosphere, I is the intensity after light passes a certain distance past the atmosphere, and $\theta$ is the solar zenith angle - - which is the angle between the normal of a given point (in this case on earth) and the light path 
incident to that point from another radiant source. AM0, AM1 and AM1.5 are the most utilized air mass standards with AM0 representing the flux of solar energy in space, AM1 for the flux of solar energy normal to the surface of earth and AM1.5 for the flux of solar energy incident to the earth at a $48.2^{\circ}$ zenith angle. AM1.5 represents the average solar radiation incident to the earth during the day and is the standard for solar research testing.The solar spectra at AM0 and AM1.5 are shown in Figure 1.6

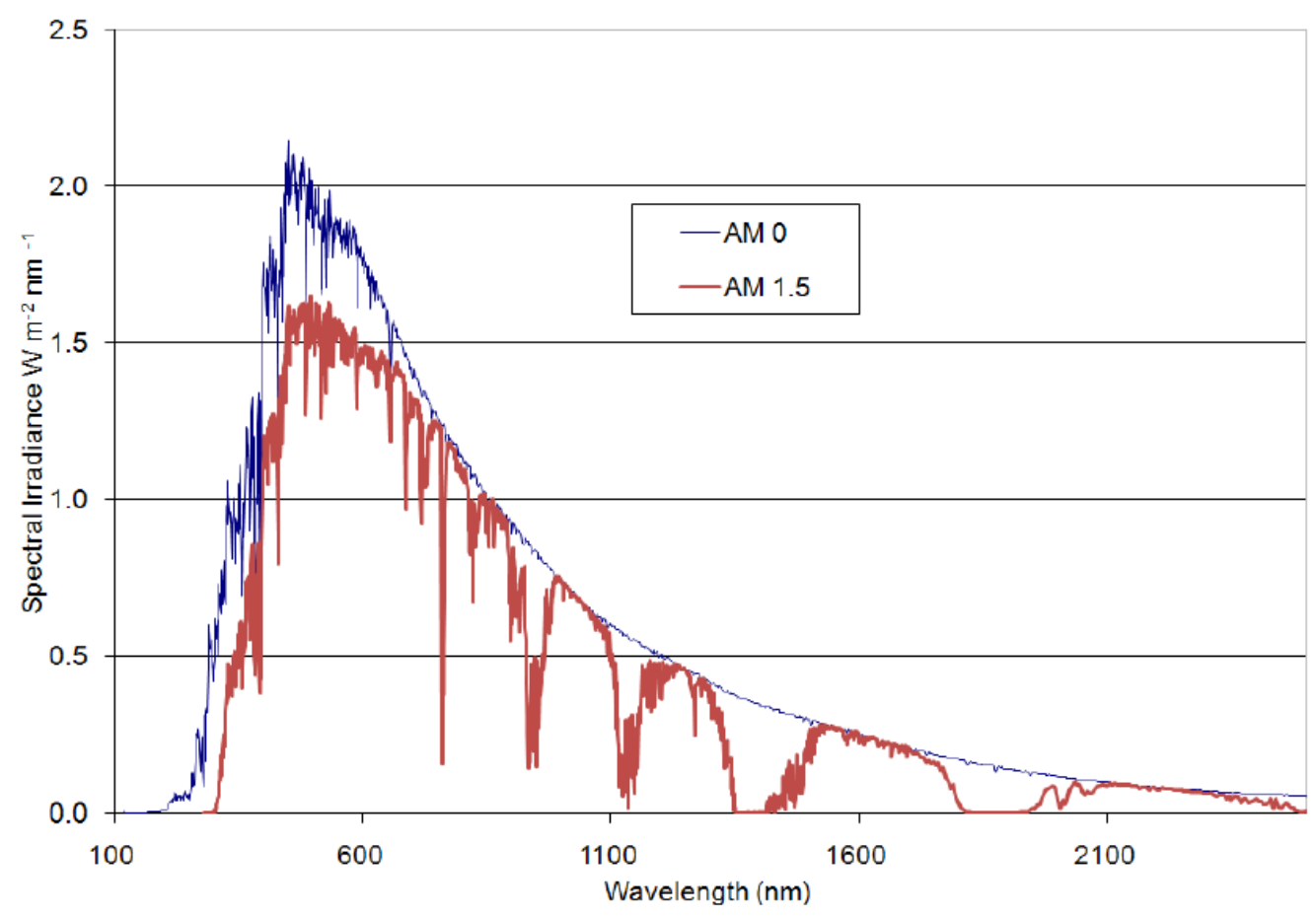

FIGURE 1 - Standard AM0 and AM1.5 solar spectra ${ }^{2}$.

Electrochemical reactions can be driven by semiconductors using energy absorbed from light. One example of this is photolysis, where semiconductors are utilized with energy from the sun to break down waste. Another area where semiconductors can be used to drive an electrochemical reaction is with the photoelectrochemical splitting of water in order to produce hydrogen fuel and much research is being devoted to this technology. 
A basic PEC cell will consist of an anode and a cathode. When the anode or cathode contains a semiconductor capable of absorbing light, it is referred to as the photo-anode or photo-cathode respectively. Photo-anodes capable of driving the water oxidation reaction will be N-type semiconductors while photo-cathodes capable of driving the water reduction reaction will be P-type semiconductors. (further explanation of semiconductors is found later in the text). When a single semiconductor is being considered in a PEC cell, it is designated the working electrode (WE), and a noble metal is utilized as the counter electrode (CE). Often, a third reference electrode (RE) is utilized to ensure proper analysis of the performance of a semiconductor in PEC testing. With two-electrode PEC testing, bias is applied between the WE and CE by a potentiostat, but how this bias is distributed is unknown. By utilizing a three electrode set-up, a bias is applied to the WE relative to the RE. Therefore, the potential at the WE can be known while the bias at the CE varies. The most common RE used for PEC water splitting is $\mathrm{Ag} / \mathrm{AgCl}$. A basic three-electrode setup is illustrated in Figure 2. With illumination, electrons are generated and are transported from photo-anode to the cathode, where they drive the water reduction reaction. Holes drive the water oxidation reaction on the surface of the photo-anode. 


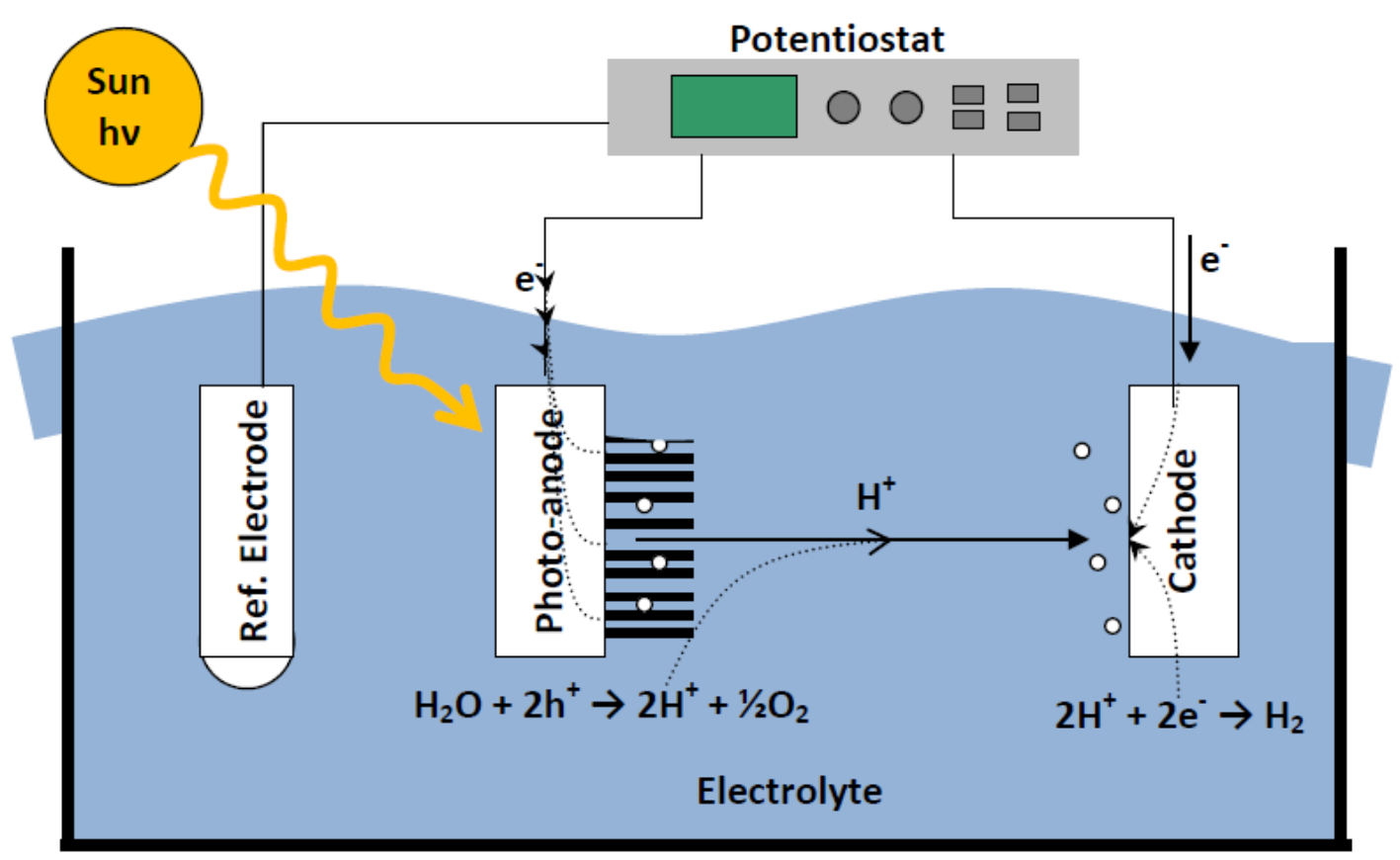

FIGURE 2 - A basic three-electrode photoelectrochemical cell

\section{Principles of Semiconductors:}

Semiconductors have two different types of conductivity referred to as N-type or P-type. These conductivity types are determined by the position of the semiconductor's Fermi band level and are representative of the amount of carriers available in the semiconductor. One of the defining characteristics of a semiconductor is the band gap $\left(E_{g}\right)$, which is the energy difference between the conduction $\left(E_{c}\right)$ and valence $\left(E_{v}\right)$ bands in the semiconductor, shown in Figure 3. When an electron is energized from the highest occupied molecular orbital (HOMO), which in the ideal case in a semiconductor would be located at the valence band, to the lowest unoccupied molecular orbital (LUMO) or conduction band in the ideal semiconductor, a hole is left behind thus creating what is referred to as an electron hole pair (ehp). Both the electron and hole of this electron-hole 
pair is able to freely mitigate its way through the crystal lattice along $E_{c}$ and $E_{v}$ respectively in opposite directions. In a semiconductor with a direct band-gap, any energy input above the $\mathrm{E}_{\mathrm{g}}$ energy will directly promote an electron to the LUMO state creating an ehp. In the case of an indirect band-gap material however, an energy equal to $\mathrm{E}_{\mathrm{g}}$ must be applied, but the bands are also separated by a certain momentum (p), which can only be overcome by providing a phonon, which is a measure of crystal vibration usually provided which can be induced by thermal energy. These bands exhibit a parabolic nature between the crystal momentum (k) and energy (E) as shown in the following expression.

$$
E-E_{c} \text { or } E v-E=\frac{p^{2}}{2 m}=\frac{h^{2} k^{2}}{2 m}
$$

With $\hbar$ being Dirac constant or reduced Planck constant $(\hbar=\mathrm{h} / 2 \pi)$ and the mass of the particle being represented by $\mathrm{m}\left(\mathrm{m}_{\mathrm{e}}{ }^{*}\right.$ for electrons in $\mathrm{E}_{\mathrm{c}}$ and $\mathrm{m}_{\mathrm{h}}{ }^{*}$ for holes in $\left.\mathrm{E}_{\mathrm{v}}\right)$. A free particle is represented in equation (6), however, complexities arise in an actual crystal due to the reduced degrees of freedom caused by interaction with atoms in the crystal structure, inhibiting direction the particle might take. Figures $4 \mathrm{a}$ and $4 \mathrm{~b}$ show the theoretical E vs. $\mathrm{k}$ band diagram for a direct band gap semiconductor and indirect band gap semiconductor material. Note that the minimum change in $\mathrm{E}$ between $\mathrm{E}_{\mathrm{c}}$ (top band) and $\mathrm{E}_{\mathrm{v}}$ (bottom band) occurs at $\mathrm{k}=0$ for direct band gap semiconductors while this minimum change in $\mathrm{E}$ occurs with a change in $\mathrm{k}$ in indirect band gap semiconductors. One example of an important material with a direct band gap is GaAs. While a phonon is required to create an ehp at the minimum $\mathrm{E}_{\mathrm{g}}$ in an indirect band gap material, if an 
amount of energy equal or greater than the energy gap at $\mathrm{k}=0$ in an indirect material is provided, an electron can be directly promoted to the conduction band. Possibly the most renowned indirect band gap material is silicon, whose indirect band gap nature has not inhibited its success in the electronics and photovoltaics industries. Due to indirect band gap semiconductors requiring a phonon and photon to absorb simultaneously to promote an electron into the band gap, light must penetrate further, requiring indirect band gap materials to be created in thicker layers. This is why the typical solar cell made from silicon must be hundreds of microns thick while a solar cell made from GaAs will only require under 10 microns of thickness to absorb incoming light.

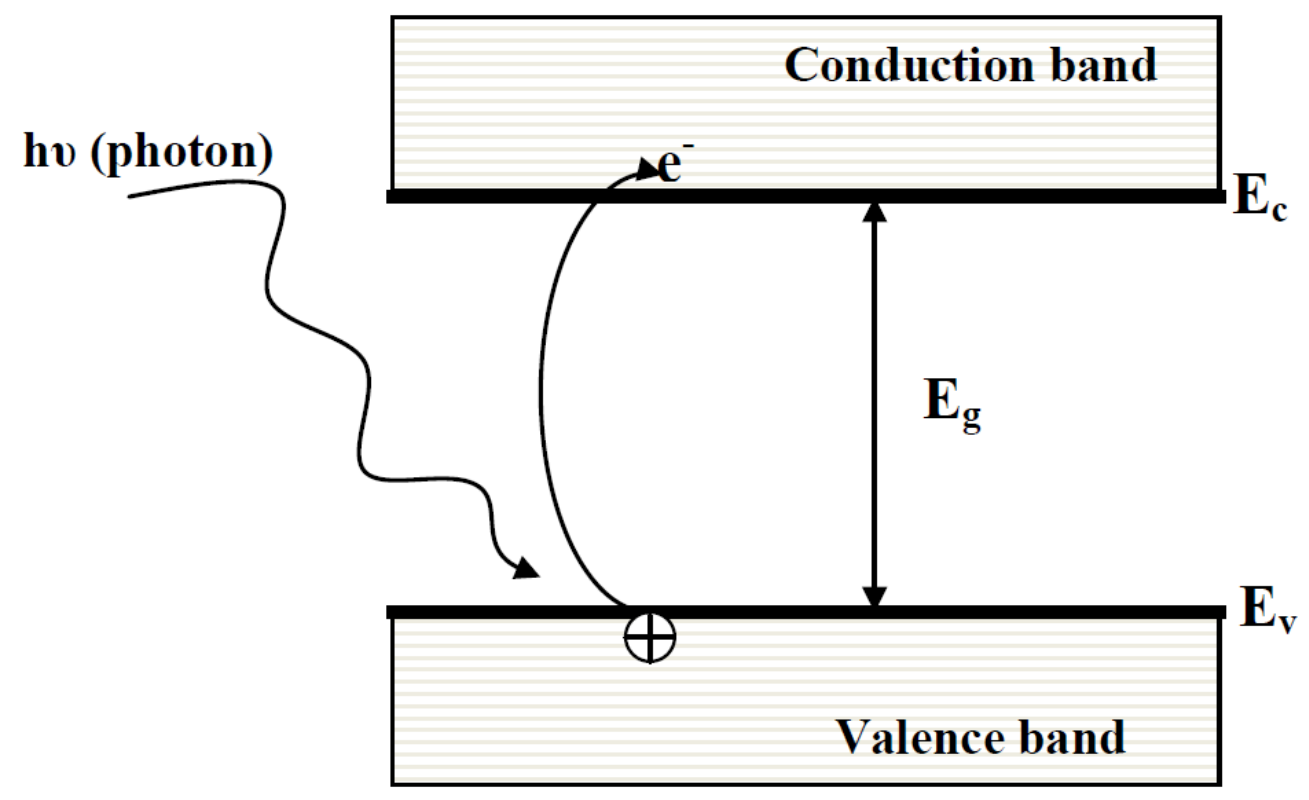

FIGURE 3 - An illustration of an electron hole pair being created as an electron is promoted from to the conduction band from the valence band. 

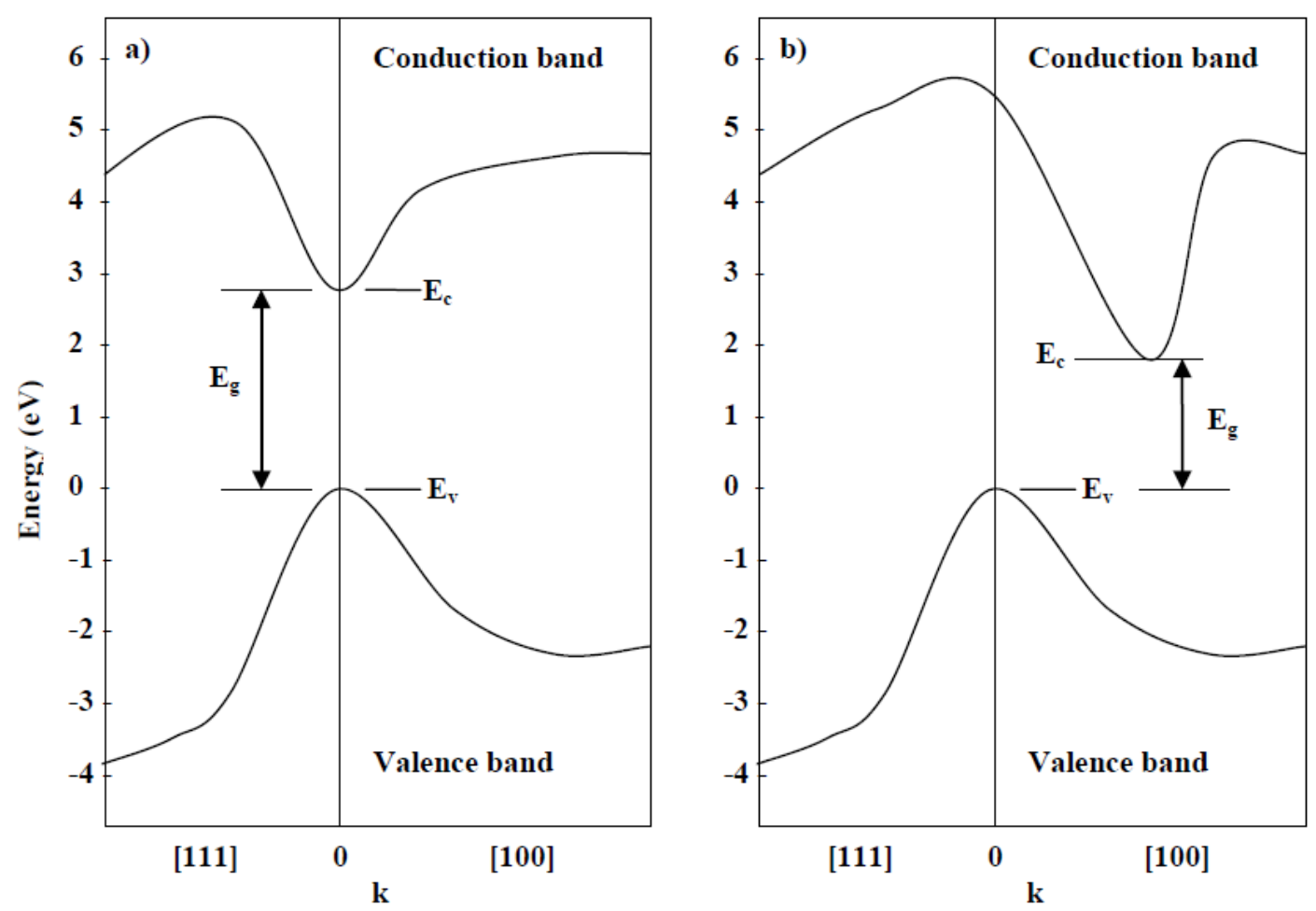

FIGURE 4 - A diagram of Energy (E) vs. crystal momentum (k) for direct (a) and indirect (b) band gap semiconductors.

The band gap represents a gap of states between the conduction and valence bands which cannot be occupied, above and below the band gap however there is an innumerable density of states which can be occupied. The Fermi-dirac distribution function takes these states into account.

$$
\frac{N(E)}{g(E)}=f_{F}(E)=\frac{1}{1+\exp \left(\frac{E-E_{F}}{k T}\right)}
$$


With $\mathrm{T}$ being the temperature in Kelvin, $\mathrm{k}$ being the Boltzmann constant, $\mathrm{N}(\mathrm{E})$

representing the amount of individual particles (electrons in this case) per energy unit per volume, and $\mathrm{g}(\mathrm{E})$ representing the quantum state density as number of quantum states per energy unit per volume.

The Fermi Level represents a level of energy whose chance of being occupied is $50 \%$, even though the Fermi level is a state that is not able to be occupied. Thus, at the Fermi level, the Fermi-Dirac distribution equals 50\%. The Fermi level is also referred to as the chemical potential $(\mu)$ or electrochemical potential of electrons when discussed in physics or electrochemistry respectively. The Fermi level in redox coupling is yielded by:

$$
E_{F, \text { redox }}=\bar{\mu}_{e, \text { redox }}=\mu_{\text {redox }}^{\circ}+k T \ln \left(\frac{c_{o x}}{c_{\text {red }}}\right)
$$

With $\mathrm{c}_{\mathrm{red}}$ and $\mathrm{c}_{\mathrm{ox}}$ representing oxidized and reduced species concentrations. Donor or acceptor states present in a semiconductor material can change the Fermi level, defining the type of conductivity that the semiconductor exhibits (n-type or p-type). The Fermi level occurs directly at the midgap state between the valence and conduction bands in materials that are naturally semiconductors, like undoped silicon. Doping impurities into the semiconductor crystal lattice can donate holes or electrons providing donor or acceptor states to change the Fermi level. One example is introducting phosphorous into a silicon lattice to shift the Fermi level in silicon towards n-type. This is because there are five valence electrons in phosphorous while silicon only contains four, thus phosphorous provides an extra electron per atom to the crystal. Conversely, Boron, with 
three valence electrons, one fewer than silicon, can be introduced into the silicon lattice in order to lower the electron count in the crystal lattice, providing more holes and shifting silicon towards p-type. On average, $10^{15}$ to $10^{19}$ impurity atoms per $\mathrm{cm}^{3}$ are needed to effectively dope a crystal n-type or $\mathrm{p}$-type. By providing this doping to a semiconductor, charge carriers become distributed differently in the semiconductor causing the density of occupied states above the the conduction band to be increased in ntype semiconductors, thus raising the Fermi level closer to the conduction band. Oppositely in p-type doping, the Fermi level shifts toward the valence band. An illustration of this is shown in figure 5.
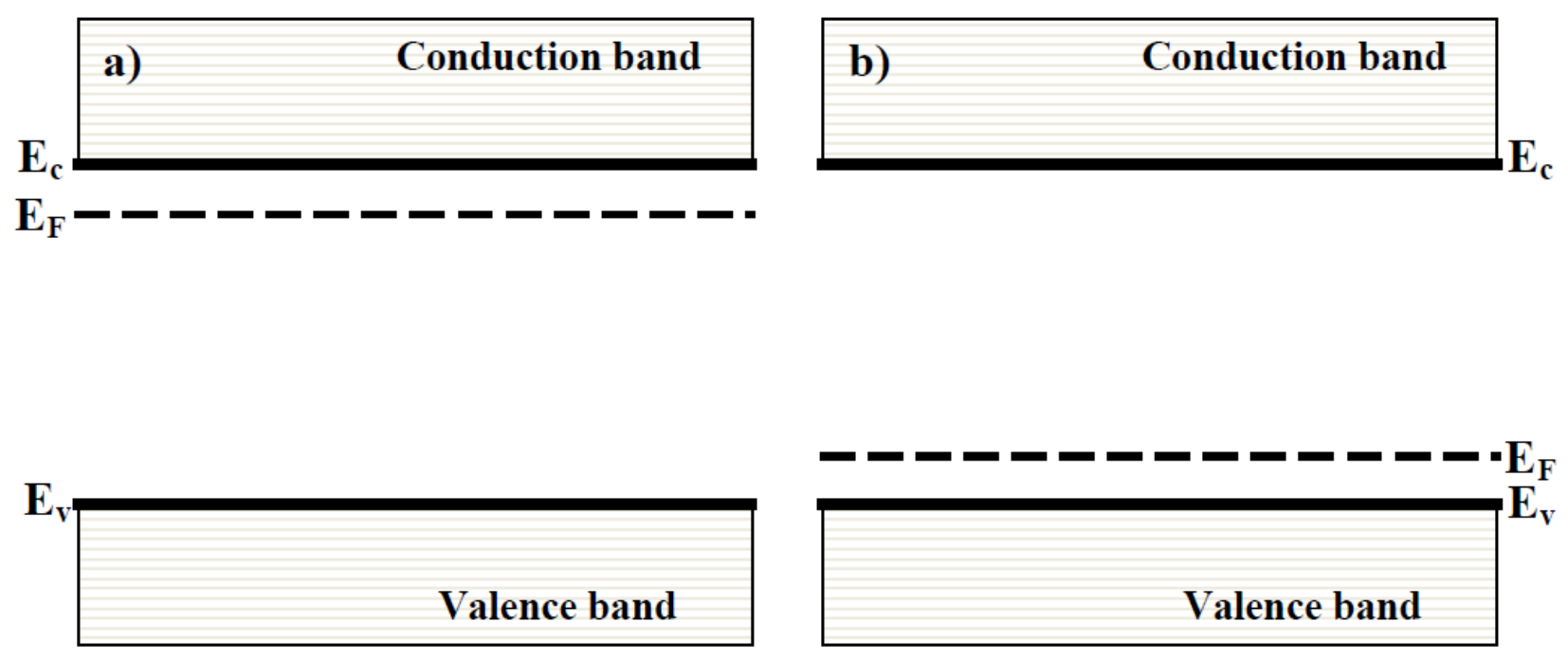

FIGURE 5 - A schematic showing how the Fermi level is shifted in n-type (5a) and ptype $(5 b)$ semiconductors.

When an electrolyte is present, equilibriation occurs between the Fermi levels of the semiconductor and of the electrolyte ${ }^{3,4}$. Figure 6 displays a photoanode/metal cathode two electrode setup under illumination, in the dark and before contact for several 
scenarios. In figure 6, voltage is plotted vs. a spatial dimension on the electrochemical scale, where the positive direction on the voltage scale is actually a negative voltage and 0 is the voltage of the normal hydrogen electrode. Thus, a rise on the band diagram actually refers to a potential shift in the negative direction and vice versa. In electrolytes, the Fermi level follows a Gaussian distribution function of redox states which depends on concentration that falls somewhere between the $\mathrm{O}_{2}$ and $\mathrm{H}_{2}$ redox couple, for extensively reviewed in several articles $s^{3,5-7}$. For n-type configurations, the semiconductor shows a greater chemical potential than in the electrolyte. This allows electron diffusion to the solution from the semiconductor surface. At the semiconductor and electrolyte surfaces, positively and negatively charged ions respectively congregate. This in turn creates a space charge region on the semiconductor side of the interface and an electrical double layer on the electrolyte side referred to as the Helmholtz layer. This translates to a bending of $E_{c}$ and $E_{v}$ on the band diagram for this system. Due to the Helmholtz layer, the position of the band edges at the interface are static and don't change during semiconductor-electrolyte interaction. This pinning is unique to each individual semiconductor-electrolyte system. There is no interaction between the semiconductor bulk and the Helmholtz layer however, and as a result the electrochemical potential can be redistributed thus causing a shift in the Fermi level, which is a property of the bulk material. The Fermi level, conduction band and valence bands each remain constant as functions of the material, each shifting congruently together. There is an electric field contained in the space charge region where the bands bend, which has a direction relative to the position of the electrolyte redox potential compared to the Fermi level. N-type semiconductors have more positive or downward shifting bands in order to reach 
equilibrium and consequently create a field that drives the majority carrier electrons away from the interface while oxidation is performed by holes driven into the electrolyte solution. P-type semiconductors have more negative or upward shifting bands which creates an electric field where electrons are driven into the solution and holes sent away from the interface. Due to this, water splitting at the photocatalyst is a process driven by minority carriers. The counter electrode material will have a far greater density of carriers and as such will be able to match the Fermi level of the semiconductor as it shifts. Charges generated by illumination oppose the electric field located in the space charge region which flattens the bands according to the degree of illumination intensity. At a certain level of illumination, the Fermi level of the semiconductor will be equal to that of the flat band potential $\left(\mathrm{U}_{\mathrm{fb}}\right)$. The flat band potential refers to the maximum possible Fermi level in p-type semiconductors without applying an external bias or minimum possible Fermi level in n-type semiconductors. Figures 6a through $6 \mathrm{~d}$ show an n-type semiconductor incapable of splitting water without applying an external bias. This is because the semiconductor's flat band potential is not above the hydrogen evolution potential. Figure 6a shows the Fermi level of the metal aligned with the electrolyte solution's Fermi level; however, no contact has been made between the two. In figure $6 \mathrm{~b}$, the electrodes have been placed in electrolyte and connected. Note that the semiconductor Fermi level lines up with the Fermi level of the electrolyte and due to the band edges being pinned, a downward bend occurs in the bulk bands. In figure 6c, illumination is provided and carriers are generated, counteracting the electric field contained in the space charge region, bending of the bands to be reduced. Despite electrons moving throughout the bulk and going to the metal, there is still not enough 
energy to drive the hydrogen evolution reaction. Thus in figure $6 \mathrm{~d}$ a positive external bias $\left(\mathrm{E}_{\mathrm{b}}\right)$ is applied which is distributed between the two electrodes. This causes a raising of the metal Fermi level and a lowering of the semiconductor Fermi level and with enough bias can drive the water reduction reaction. When a p-type semiconductor has too high of a flat band potential, a negative external bias can be applied in order to lower the Fermi level of the metal and raise that of the semiconductor. A photoanode with ideal properties for driving electrolysis by itself is shown in figure 6e. 

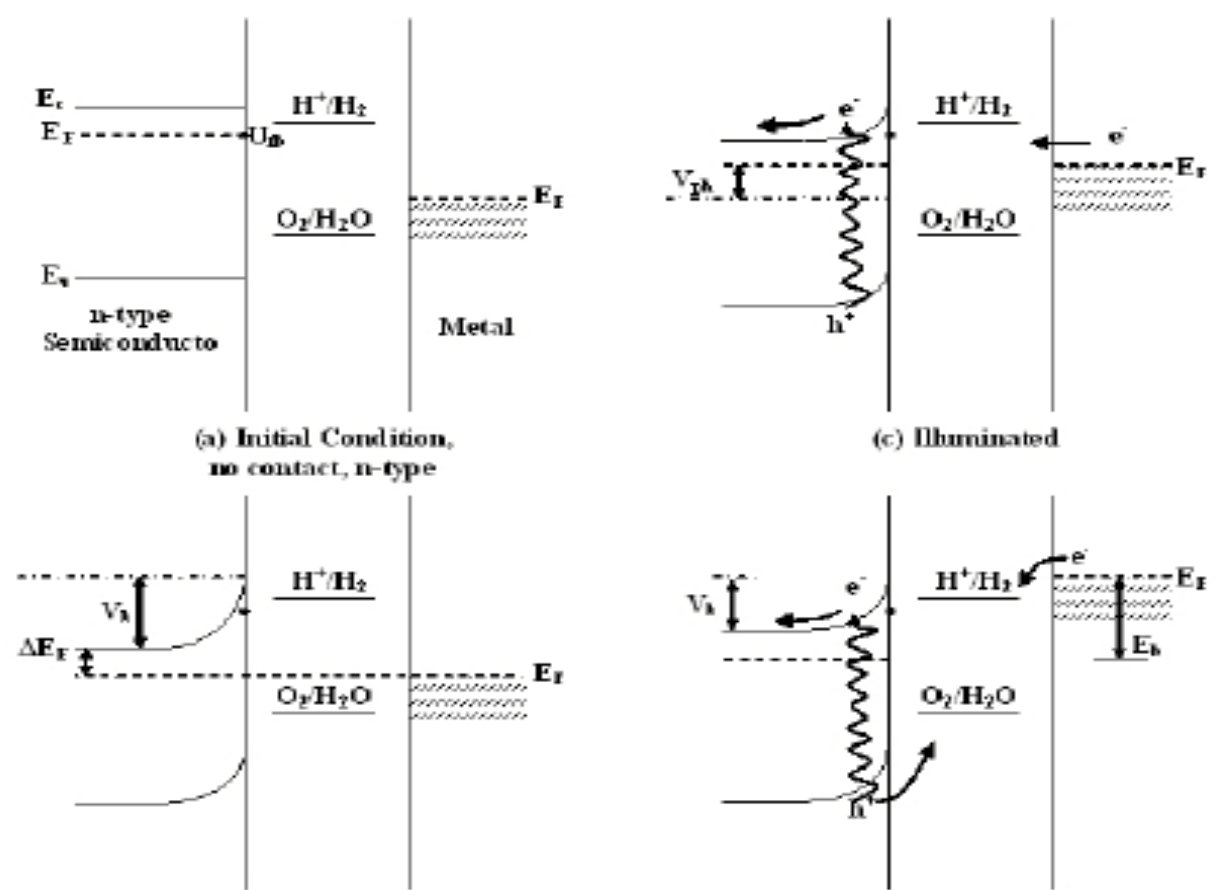

(c) Hbunimated

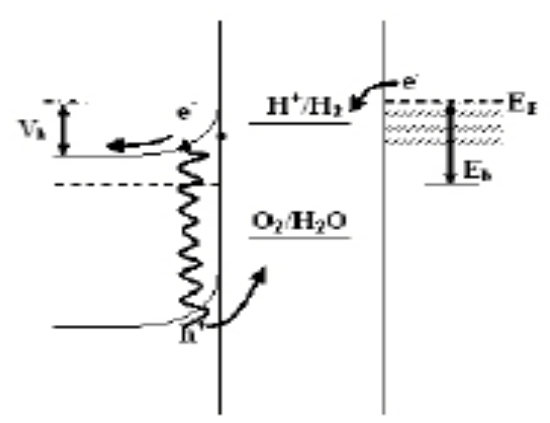

(b) Equihib rimn.

Dauk

(d) Hlmointed,

with Bias
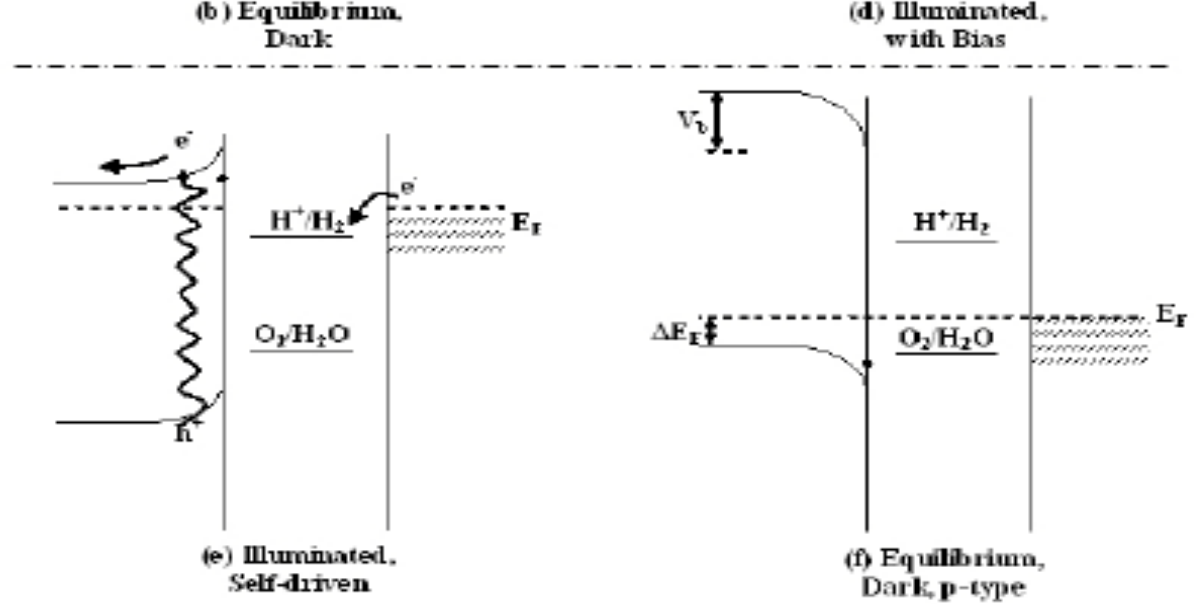

FIGURE 6 - Band diagrams for n-type and p-type semiconductors in electrolyte for water splitting. Figure (a) shows an n-type semiconductor before contact with a cathode or electrolyte. Figure (b) shows the n-type semiconductor in the dark at equilibrium with the electrolyte and cathode. Figure (c) shows the same set-up as in (b) under illumination and Figure (d) shows the same with an external positive bias. Figure (e) shows the ideal n-type semiconductor that can split water without external bias and Figure (f) shows the p-type correlate of figure (b). 


\section{Requirements for a viable PEC material:}

The criteria for a viable material that is able to perform water splitting efficiently and with proper stability are known and listed below ${ }^{8}$.

1) The material must exhibit a Band gap that approximately falls between $1.7 \mathrm{eV}$ to $2.2 \mathrm{eV}$.

2) Must have substantial charge carrier conductivity and reaction kinetics.

3) The energy of the HOMO must be below the oxygen evolution potential and the LUMO energy must be above the hydrogen evolution potential.

4) Must show stability in aqueous solution.

5) Must be an abundant earth resource, inexpensive and producible on the mass scale.

With a minimum energy of $1.23 \mathrm{eV}$ needed to perform electrolysis, thermodynamic losses and reaction kinetics to take into account, it is expected atleast a $1.7 \mathrm{eV}$ band gap is required. This band gap needs to be limited to below $2.2 \mathrm{eV}$ however in order to insure enough of the solar spectrum is absorbed in order to create electron hole pairs in the material. Secondly, because the semiconductor is performing a reaction at the surface, the charge transfer kinetics between the semiconductor surface and electrolye need to be faster than the kinetics of the decomposition reaction. For example, $\mathrm{ZnO}$ works well as a photocathode because the potential at which water reduction occurs is greater than the corrosion reduction potential that occurs in $\mathrm{ZnO}^{9}$. Third, in order to carry forth the water splitting reaction, $\mathrm{E}_{\mathrm{v}}$ must be a higher energy than that of the water oxidation potential, 
which occurs at $1.23 \mathrm{eV}$ vs. NHE, and $\mathrm{E}_{\mathrm{c}}$ must be a lesser energy than that of the water reduction potential that occurs at $0 \mathrm{eV}$ vs. NHE. As for the fourth requirement, not all semiconductors will be stable under aqueous conditions and since the goal is to split water, a semiconductor that does not undergo degradation in aqueous solution during operation needs to be chosen. Last, the material chosen needs to be economically practical and available on the mass scale. While an expensive PEC water splitting cell composed of precious metals may work great on the international space station, it will be economically out of reach for the majority of Earth's population.

\section{PEC Efficiency}

Currently, the most efficient PEC water splitting cell in terms of photoconversion was achieved by Turner et al at NREL using a III-V monolithic $\mathrm{GaInP}_{2} / \mathrm{GaAs}$ tandem cell exhibiting a $12.4 \%$ efficiency $^{10}$. The major drawback to this cell is that it easily corroded over a short time frame. The efficiency of photoconversion is also referred to as the solar to hydrogen conversion efficiency and represents the total light that is converted to energy divided by the total incoming light energy as shown in the following equation ${ }^{11}$,

$$
\varepsilon=\frac{1.229 I_{P}}{P_{t}}
$$

Where $P_{t}$ is the light irradiance (or input power) in $W / m^{2}, I_{p}$ represents the photocurrent in $\mathrm{A} / \mathrm{m}^{2}$, and 1.229 represents a constant in volts that is derived from taking the quotient of the Gibbs free energy of the hydrogen evolution reaction under standard pressure and temperature conditions by the amount of electron moles used to create 1 mole of $\mathrm{H}_{2}$ and 
by Faraday's constant. This equation assumes that all carriers created are used for evolving oxygen and hydrogen and as such a contradiction occurs when power output comes from degradation of the photoelectrode through corrosion. Equation (9) also assumes that the photocurrent is generated without an external applied bias. While the most desirable case for a photoelectrode is to spontaneously perform PEC water splitting upon illumination; most often it is apply an external bias to promote the most photocurrent output. Equation (9) can be modified in order to take this external bias into account as shown below.

$$
\varepsilon=\frac{\left(1.229-V_{\text {bias }}\right) I_{P}}{P_{t}}
$$

With $\mathrm{V}_{\text {bias }}$ representing the applied external bias that occurs as a difference in potential between the photoelectrode (WE) and the counter electrode. Preferably, efficiency would be determined using a two-electrode set-up because in a three electrode set-up, $\mathrm{V}_{\text {bias }}$ cannot be measured by the potentiostat and an external voltmeter is required to measure the $\mathrm{V}_{\text {bias. }}$ 


\section{Previous studies on PEC materials}

There are very strict criteria for a semiconductor to be considered for PEC water splitting. So much so that there are very few materials that even meet basic requirements such as band gap, position of band edges and stability. There are two existing routes that can be taken in order to find the model semiconductor capable of splitting water; by synthesizing it from the near limitless amount of element combinations or by making modifications to materials that have already been considered. Since so many semiconductors have already been studied, an attempt to overcome the inherent weaknesses or amplify the strengths of these materials could lead to a semiconductor capable of performing PEC water splitting. The materials and strategies being researched for PEC water splitting can be broken down into three distinct categories:

\section{$\checkmark$ Metal Oxides}

\section{$\checkmark$ Elements from Group IV and III-V elemental combinations $\checkmark$ Others}

Work currently has been mostly done on binary and ternary elemental combinations and as such, any higher order combinations won't be discussed further in this writing due to the small amount of research available.

Metal Oxides: The original discovery of the water splitting reaction occurred in 1972 using Titania $\left(\mathrm{TiO}_{2}\right)$ and has been extensively studied since that point ${ }^{1,12-21}$. Titania has 
many great qualities for performing PEC electrolysis such as excellent stability in aqueous solution over a wide $\mathrm{pH}$ range, it's conduction and valence bands straddle the water oxidation/reduction bands and it is naturally an n-type photoanode. However, Titania's band gap is $3 \mathrm{eV}$ and as such, isn't capable of absorbing a large amount of solar energy which severely limits the photoconversion efficiency titania can exhibit. Most of the research done on the Titania system has been in attempting to lower the band gap of Titania or finding ways to mitigate the band gap requirement. The major problem with trying to decrease Titania's band gap is that it has been shown that one of the reasons Titania is so stable is due to its wide band gap and ask it is decreased, degradation of the semiconductor increased $^{22}$. There have been many other promising metal oxides but have all fallen short due to having to large of a band gap. Some of the more noteworthy metal oxides are $\mathrm{WO}_{3}{ }^{23-26}, \mathrm{ZnO}^{27-29}, \mathrm{SrTiO}_{3}{ }^{30-33}, \mathrm{SnO}_{2}, \mathrm{Ta}_{2} \mathrm{O}_{5}, \mathrm{KTaO}_{3}$ and $\mathrm{ZrO}_{2}{ }^{6}$. In order to mitigate the band gap requirements, some studies have focused on changing other properties of the material, such as increasing the surface area. One approach to this is to use single dimension nanostructures, discussed later in this writing.

One very exciting metal oxide to the PEC water splitting community is Iron Oxide. Three main types of iron oxide exist including iron(II) oxide (FeO), naturally occurring iron (II,III) oxide, also called magnetite $\left(\mathrm{Fe}_{2} \mathrm{O}_{4}\right)$, , and iron (III) oxide, also called hematite $\left(\mathrm{Fe}_{2} \mathrm{O}_{3}\right)$. Magnetite is not eligible for use in water splitting due to it's $0.1 \mathrm{eV}$ band gap, well under that required for water splitting. On the opposite spectrum, FeO has a $2.5 \mathrm{eV}$ band gap, too large to be appropriate for water splitting. Also to be mentioned is maghemite, the gamma phase of $\mathrm{Fe}_{2} \mathrm{O}_{3}$. It has a close to ideal band gap of $2.03 \mathrm{eV}$ but unfortunately is highly ferromagnetic, making it a poor candidate for a PEC electrode. 
However, hematite, the alpha phase of $\mathrm{Fe}_{2} \mathrm{O}_{3}$, appears to be the perfect candidate due to its many desired properties ${ }^{34-71}$. Naturally, $\alpha-\mathrm{Fe}_{2} \mathrm{O}_{3}$ is an n-type semiconductor with an indirect band gap of $2.2 \mathrm{eV}$ which is close to the upper limit of the ideal band gap range allowing absorption of a large amount of the solar spectrum. It also shows great stability under aqueous conditions at any $\mathrm{pH}$ above 3 . Not to mention, it is basically rust and as such is composed of two of the most abundant materials on earth, is not a burden on the environment, extremely cheap and should be relatively easy to manufacture. Given all of these advantages, there are some major hindrances to hematite's success as a PEC water splitting material. First, hematite acts as a Mott-insulator, meaning that it poorly transports charge, even though it facilitates charge separation ${ }^{52,57,63}$. Also, because it is generally is synthesized as a polycrystalline film with many grain boundaries and unordered oxygen vacancies throughout the crystal, electron hole pairs quickly recombine at a high rate $^{72}$. Hematite also has anisotropic conductivity along the (001) plane, which means it conducts better across the (001) plane than in the [001] direction by four orders of magnitude ${ }^{40,63,67}$, which again causes problems in polycrystalline morphologies. Lastly, the edge of hematite's conductive band is lower than the hydrogen evolution potential on the electrochemical scale by $2 \mathrm{eV}$ (or $.2 \mathrm{eV}$ more positive) ${ }^{73}$. Though hematite has its problems, it seems modification of the material to be able to perform PEC water splitting is feasible. Doping and alloying of the material using Si 34,41,44,69$, \mathrm{Ti}^{34,35,61}, \mathrm{Pt}^{36}, \mathrm{Mg}^{37,55,74}, \mathrm{Ge}^{44}, \mathrm{Zn}^{39,49,75,76}, \mathrm{Nb}^{73,77}, \mathrm{Ta}^{78}, \mathrm{Mo}^{48}, \mathrm{Cr} \mathrm{Cu}^{38}$, and $\mathrm{Ti}-\mathrm{Al}^{79}$ have all been successfully performed and have yielded significant improvements. Surface modification using Fluorine ${ }^{35}$ and $\mathrm{Co}^{41}$ has also been studied. Of these modifications to hematite, the best enhancement has come from introducing $\mathrm{Si}$ and $\mathrm{Ti}$ 
into the crystal structure which increases the concentrations of donors, thus improving conductivity. Si also reduces the crystal size, improves crystallinity and effects orientation of the basal plane (001). Surface modification using fluorine lowered the conduction band edge energy and Cobalt helped to catalyze the water oxidation reaction. Changing morphology and improving crystallinity also helps to reduce and eliminate some limitations. The use of nanorod and nanowire structures aids in reducing the limitations of hematite and is discussed later in the text.

Hematite samples have been synthesized using many different methods such as sol-gel ${ }^{80-}$ ${ }^{84}$, single crystal ${ }^{85,86}$, physical vapor deposition (PVD) ${ }^{87}$, ceramic processing ${ }^{78}$, aqueous self-assembly ${ }^{8,89}$, chemical vapor deposition (CVD) ${ }^{14,41,52,90,91}$, thermal oxidation ${ }^{92-94}$, plasma oxidation $^{95-97}$, electrochemical deposition ${ }^{36,48,98-100}$ and spray pyrolysis ${ }^{37,38,50,75,98}$. The product of the majority of these methods is a polycrystalline film, which as previously discussed, has inherent disadvantages. Many of these methods are also not practical for scaling up to a manufacturer level. Thermal and plasma oxidation can both reliably reproduce high quality nanostructures and have the ability integrated into a large scale synthesis process. Plasma oxidation has the advantage of being able to produce these nanostructures must faster and may have other inherent advantages, as discussed later in the text.

\section{Elements from Group IV and III-V elemental combinations}

Many binary and ternary combinations of elements from group IV and groups III-V exist that have promising properties as semiconductors. The major advantage of materials 
from these groups is that they are fairly easy to synthesize in pure single crystalline form by means of metal organic CVD that give high photoconversion efficiencies. The big disadvantage of these materials is that they have poor stability and their band edges don't straddle the oxygen evolution reaction, save one III-V combination. The bands of each III-V binary material are aligned to evolve hydrogen, however the only III-V capable of driving the oxygen evolution reaction is $\mathrm{GaN}^{101,102}$. The band gap of $\mathrm{GaN}$ is far too wide at $3.4 \mathrm{eV}$, however. Silicon carbide, a group IV binary semiconductor, also has bands properly aligned in order to split water and is stable in aqueous conditions, but also has too wide of a band gap at $3.0 \mathrm{eV}^{103}$. To top it off, to produce these high quality single crystal films require the use of advanced equipment with high processing costs. Ternary and higher order materials can be created using group III and V elements to produce useful properties. Often, a third element can be introduced in order to properties somewhere in between two semiconductors. One case of this is combining a low band gap material, such as InN with a $.7 \mathrm{eV}$ band gap, with a high band gap material, such as $\mathrm{GaN}$ in order to lower the band gap. Depending on composition, the band gap is lowered to a similar degree ${ }^{104-107}$. Other semiconductor systems have been researched for PEC water splitting such as GaPN, GaAsPN, GaInPN ${ }^{108,109}$, and $\mathrm{GaInP}_{2}{ }^{110-112}$. GaAsPN degraded quickly. GaP ${ }_{98} \mathrm{~N}_{.02}$ had a promising $2 \mathrm{eV}$ band gap, however introducing nitrogen into the crystal caused lattice mismatches with the GaP layer underneath in turn causing a significant reduction in efficiency, but yielded reduced vulnerability against corrosion. Introducing indium at different ratios to form $\mathrm{Ga}_{.95} \operatorname{In}_{.05} \mathrm{P}_{.975} \mathrm{~N}_{.025}$ and $\mathrm{Ga}_{962} \mathrm{In}_{.038} \mathrm{P} .{ }_{976} \mathrm{~N}_{.024}$ fixed the lattice mismatch in these materials and still exhibited a 
direct $2 \mathrm{eV}$ band gap. These materials still unfortunately exhibited poor photoconversion efficiencies.

One way to utilize these high band gap III-V materials is to use a tandem cell, which is two or more semiconductor materials combined by connecting through voltage independent contact (ohmic). By stacking a high band gap material on top of a lower band gap material, all higher energy light is absorbed by the higher band gap semiconductor and lower energy light transmits through to the lower band gap semiconductor. Thus, a wider range of photons are absorbed allowing higher efficiencies. To date, the most efficient PEC water splitting cell that has been reported is a tandem cell. $\mathrm{p}-\mathrm{GaInP}_{2}$ was placed in tandem onto a $\mathrm{p} / \mathrm{n}$ GaAs junction to achieve a $12.4 \%$ Solar-to-hydrogen efficiency by Turner $e t$ al as discussed earlier in the text ${ }^{10}$. If it weren't for poor stability and high processing costs, this would meet the criteria for an ideal semiconductor to perform photo-electrolysis.

Others: Other potential strategies and semiconductors have been looked into for PEC water splitting applications. Some schemes such as using a particle suspension, applying protective coatings and using hybridized architectures have been tried. Materials such as the metal sulfides have also been attempted. None of these studies have shown many positive results for PEC water splitting applications. For further explanation of these pursuits please read the following review articles ${ }^{6,8,16,113-115}$. 


\section{Nanowires for PEC Water Splitting}

One popular route to improving PEC performance in existing materials is to modify the morphology. Nanowires exhibit many desirable characteristics that can aid in PEC water splitting. One advantage is that a high surface-to-volume ratio is provided giving a higher effective area for absorption as well as more surface area for reaction to take place at. Nanowires are also single crystal in nature leading to fewer grain boundaries than would be found in a polycrystalline film ${ }^{88,95,96}$, which in turn would mitigate many of the disadvantages of polycrystalline films discussed earlier in the text, such electron hole pair trap sites ${ }^{68}$. Nanorod and nanowire geometries also provides a minimal distance for minority carriers to diffuse as well providing a single orientation pathway perpendicular to the back contact without random losses as would occur in a polycrystalline or nanoparticle structure. Minimizing the distance minority carriers must diffuse can help to improve photoconversion efficiency by reducing the probability of recombination, as has been shown in hematite ${ }^{51}$. Nanowires also lend themselves to alloying and doping with other metals. As feature size approaches the Bohr radius $(\sim 5 \mathrm{~nm})$, quantum confinement can occur $^{116}$, which could lead to an upward shift (energy decrease) in the conduction energy band without significantly changing the band gap. 1-D nanostructures have demonstrated better performance than thin films and particles in several systems. $\mathrm{TiO}_{2}$ nanotubes and nanowires have been synthesized and compared to particles and thin films in several studies already ${ }^{17-19} \cdot \mathrm{TiO}_{2}$ nanowires were shown to have twice the solar to hydrogen efficiency as a thin film in one study ${ }^{19}$ and twenty times the solar-to-hydrogen efficiency as a nanoparticle film in another study ${ }^{18}$. These significant improvements in 
photocurrent are attributed to more available surface area and fewer grain boundaries. $\mathrm{ZnO}$ was also reported to exhibit twice as much photoconversion efficiency as a $\mathrm{ZnO}$ thin film ${ }^{28}$

Hematite nanorod and nanowire morphologies have been successfully synthesized in several studies using varying methods ${ }^{91,92,94-96,116-121}$. Hematite has been synthesized into nanowire arrays using oxygen RF plasma on the second time scale ${ }^{95}$. Further study of these materials have shown that the nanowires are indeed single crystal and have uniform oxygen vacancy planes repeating in the direction of growth along the basal (001) plane throughout the superstructure ${ }^{96,97}$. As discussed previously, conductivity along the basal (001) plane is higher in hematite, which could allow enhanced carrier conductivity along these oxygen vacancy planes to the back contact in hematite nanowires. These oxygen vacancies act as trap sites and are generally responsible for recombination of charge carriers, as in polycrystalline samples where they are randomly located throughout the sample. By having these oxygen vacancy planes oriented parallel to eachother, charge carriers have a direct route to conduct to the back contact without recombining with oxygen vacancy trap sites. One metaphor that can be used is to compare the parallel oxygen vacancy planes to a bowling lane. Though a gutter (oxygen vacancy trap site) is on each side of the lane, a ball can still roll down the center of the lane to strike the pins (back contact) without ever encountering the gutter. Now imagine that the gutter (trap sites) was randomly distributed as holes throughout the bowling lane, it is doubtful that even the best professional bowlers could bowl a very good game on a lane like this and helps explain why most hematite structures may exhibit poor conductivity. 
As reported previously by a student in our lab named Boris Chernomordik in his MEng thesis completed at UofL entitled, "Comparison of $\alpha-\mathrm{Fe}_{2} \mathrm{O}_{3}$ electrodes grown by direct plasma and thermal oxidation of iron for photoelectrochemical water splitting," thermal oxidation takes 5+ hours to produce nanostructures and has the side effect of also growing a mixed phase iron oxide interfacial layer that inhibits conductivity to the back contact. Boris also reported that plasma oxidation, on the other hand, takes only minutes to produce nanostructures, the interfacial layer created is 5 times thinner than that of thermal oxidation and is of less mixed phase. Most importantly, Boris showed that the nanowires synthesized by plasma oxidation under vacuum have a band gap energy of 2.1 $\mathrm{eV}$, exhibit a significant photoactivity of $255 \mu \mathrm{A} / \mathrm{cm}^{2}$ and act as an n-type semiconductor. A figure comparatively displaying the advantages of plasma oxidized nanowire arrays against thermally oxidized nanowire arrays is shown in figure 7. 


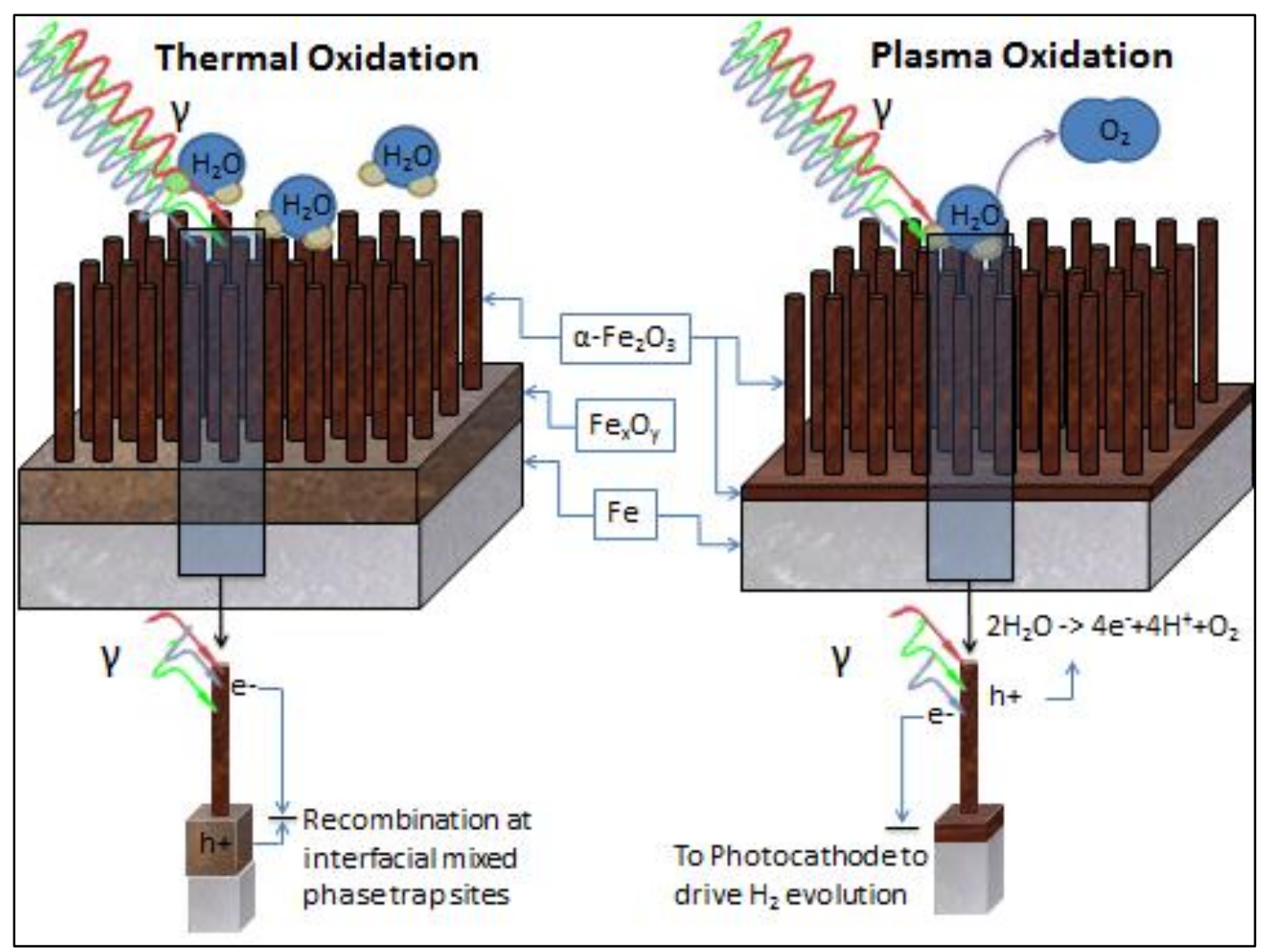

FIGURE 7 - A comparative look at the advantages of Plasma oxidized hematite nanowire arrays versus thermally oxidized nanowire arrays.

While this is a great achievement, a process that must occur under vacuum does not lend itself well to the manufacture scale and as such, ambient or atmospheric jet plasma synthesis of hematite is being researched and is the topic of this paper.

\section{Purpose and Intent of this Study}

The purpose of this study is to characterize hematite nanowire arrays synthesized by Atmospheric Jet Plasma. It is desired to understand the photoactive properties of the material and understand any differences that occur between it and its counterpart 
synthesized under vacuum. It is also desirable to understand properties of the nanowire surface and how they affect photoconductivity. 


\section{INSTRUMENTATION AND EQUIPMENT}

The instruments, equipment and procedures utilized in order to perform this study are discussed in this section. The specific conditions used for the experiment are discussed in section IV.

\section{Synthesis:}

\section{Atmospheric Microwave Plasma Jet Reactor}

An Atmospheric Microwave Plasma Jet Reactor designed and assembled in our lab was used to perform plasma oxidation of iron samples ${ }^{122}$. The reactor can be operated in two different configurations. One configuration is used process bulk powders that where the flame pulled through a long quartz tube oriented downwards, producing a 10-15 inch plasma flame and allowing powder feeding from the top. The second configuration, used in this study, orients the flame upward into the atmosphere producing a 4-5 inch plasma flame. The plasma jet exits the reactor through a 1.5 inch ID quartz tube using a concentric gas delivery system in order to swirl the gases from the quartz tube, providing a uniform plasma flame. The reactor can operate from ambient pressure ranges all the way down to a few torr and at power ranges from .3 to $3 \mathrm{~kW}$. A schematic showing the modified, upward pointing, configuration is shown in figure 8. Due to the plasma occurring at atmospheric pressures, Langmuir probes are incapable of taking accurate 
measurements and due to the novelty of this technology, no probe has been developed in order to take measurements of properties such as electron temperature in the plasma.

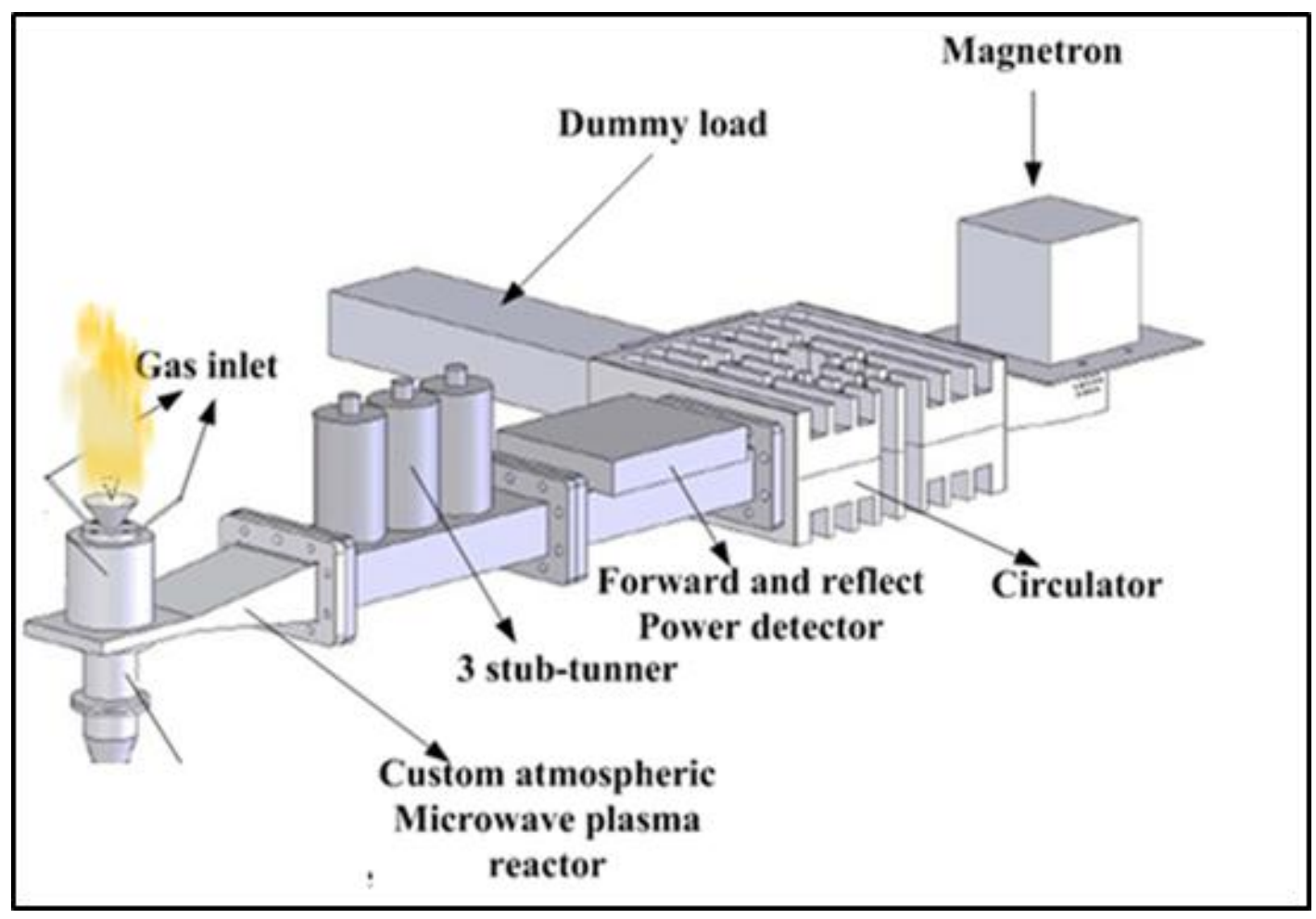

FIGURE 8 - Schematic of an Atmospheric Microwave Plasma Jet Reactor. A metal foil can be exposed to the upward directed plasma flame to perform plasma oxidation under ambient conditions.

\section{Characterization:}

\section{Photoelectrochemisty Set-up}

A three-electrode PEC cell set-up was used to determine PEC characteristics.

Synthesized photoanodes were used as the working electrode, a platinum mesh was used as the counter electrode and a $\mathrm{BAS} \mathrm{Ag} / \mathrm{AgCl}$ electrode was used for the reference 
electrode. A two compartment beaker with a quartz window was filled with $1 \mathrm{M} \mathrm{KOH}$ and the working electrode, counter electrode and reference electrode were all placed in the compartment closest to the quartz window. The two compartment design would allow collection of hydrogen and oxygen separately, but only photoactivity experiments were done in this study. This three electrode set-up is shown in Figure 9:

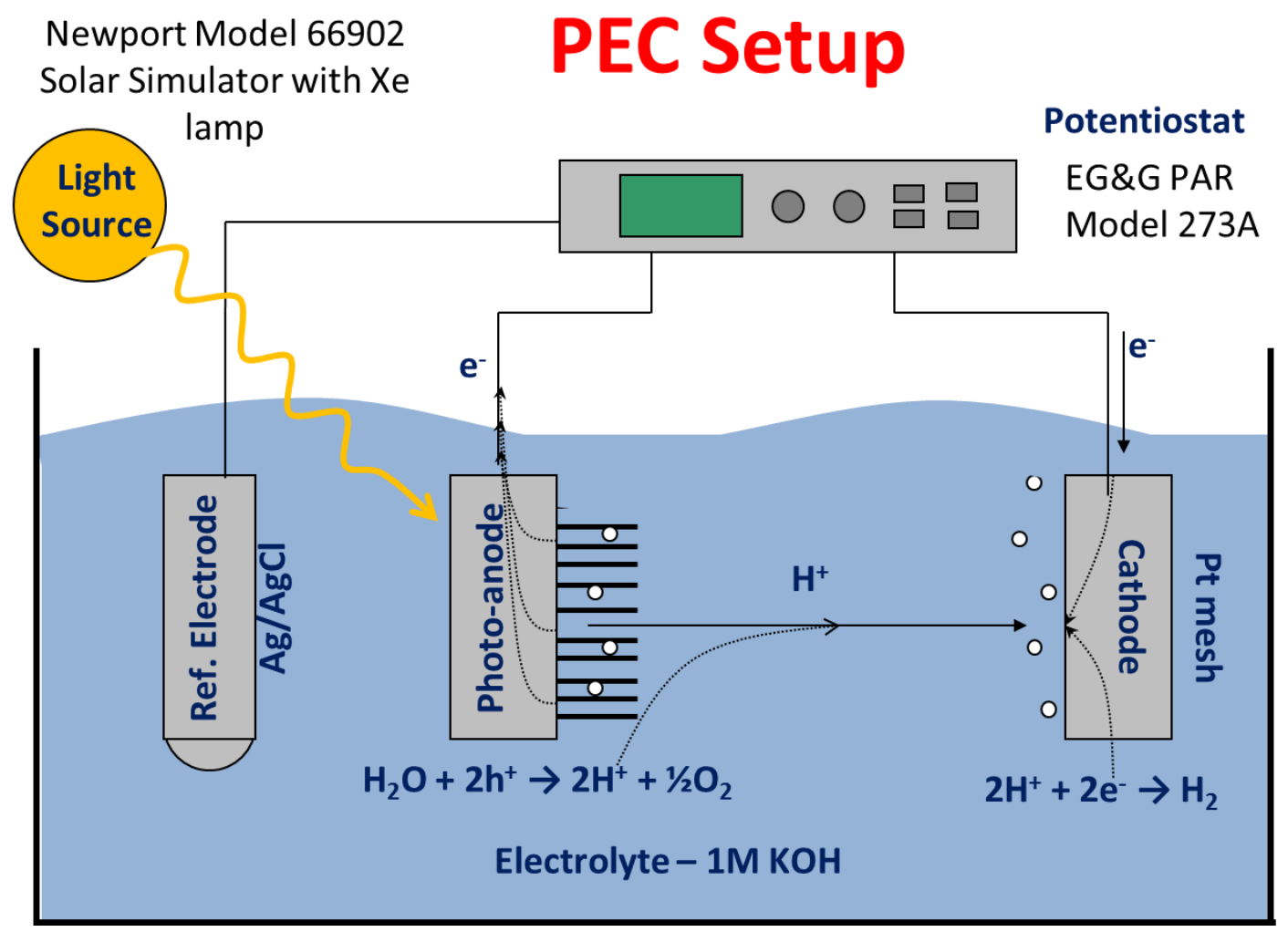

FIGURE 9 - A schematic of the typical three electrode PEC cell.

To produce illumination a Newport Instruments solar simulator with 300 Xe lamp. Light intensity calibrations were done using a Thorlabs power meter model D10MM with PM100 readout was used. To monitor/apply current and potential a Princeton Applied Research Potentiostat/Galvanostat model 273A was used that was operated by a computer using Powersuite Electrochemistry software. 


\section{Scanning Electron Microscope}

A scanning electron microscope (SEM) is primarily used for determining morphological and topographical assessment and is able to zoom in on a sample more than 500,000 times. Morphologies of synthesized samples were analyzed using a Nova 600 Nanolab SEM by FEI. The SEM works by focusing an high energy electron beam generated from a field emission gun onto the sample using electromagnetic apertures and lenses. A raster scan pattern is then performed with the electron beam by scanning coils. The beam of electrons that interacts with the sample causes a whole host of emission from the sample including X-rays, Auger electrons, backscattered electrons, secondary electrons and luminescence. All of these emissions are the result of inelastic interactions except backscattered electron emission, which is elastic, meaning energy is conserved. An in lens detector is typically used to collect secondary electrons, electron emission due to absorption of the electron beam (primary electrons), in order to image the surface topography of the sample.

\section{Transmission Electron Microscope}

Transmission Electron Microscopy is a form of microscopy that can resolve images at levels tens of thousands smaller than traditional light microscopy, enabling it to resolve images on the atomic scale. It however can only work with ultra-thin samples. It works by transmitting a high energy electron beam through an ultra-thin sample and images the 
interaction that occurs between the beam and the sample. TEM works on the de Broglie hypothesis which stated that wavelength of an electron is smaller than the wavelength of light by several orders of magnitude, thus reducing the the resolution size detectable.

Imaging for operation of the TEM is typically done on a thin (10's of micron) zinc sulphide phosphor screen and can be digitally observed using multiple electron multiplying charge-coupled devices (EM-CCD). 


\section{PROCEDURE/EXPERIMENTAL METHODS}

\section{Atmospheric MW Plasma Synthesis}

Sample Preparation: Iron samples were prepared by cutting individual sections measuring $1 \mathrm{~cm}^{2}$ from $2 \mathrm{~mm}$ thick $99.5 \%$ pure iron sheet obtained from Advent Research Materials. Native oxides were removed by first sanding down the samples using 220 grit sandpaper, treated with ethanol and dried.

Synthesis Procedure: A plasma composed of air and oxygen was created in the atmospheric microwave plasma jet reactor, described previously in section III and by Kumar et al. ${ }^{122}$, in order to oxidize the samples. While several different flow rates of air and oxygen were used, normally approximately $12 \mathrm{lpm}$ of air and $500 \mathrm{sccm}$ of oxygen were used. Argon was used to help spark the reactor flame and flow was turned off after starting the reactor. Power provided to the plasma varied from 300-1000 W. Prepared samples were then exposed to the plasma flame being held by tweezers 2-3 inches from the bottom of the flame. Exact height above the flame was varied throughout the experiment in order to control substrate temperature and exposure times ranged from two to twenty minutes. 


\section{Electrode Preparation}

In order to accurately take photoelectrochemical measurements, the electrode must be prepared in such a way to allow contact only between the semiconductor and electrolyte surfaces. The electrode must also provide an ohmic contact of minimal resistance. A procedure adopted from John Turner's group at NREL was used in order to prepare the 1 $\mathrm{cm}^{2}$ hematite samples for PEC measurements. Detailed instruction of these procedures for preparing electrodes is provided in Appendix A. Figure 10 shows a typical electrode before assembly and before and after coating with insulating epoxy.

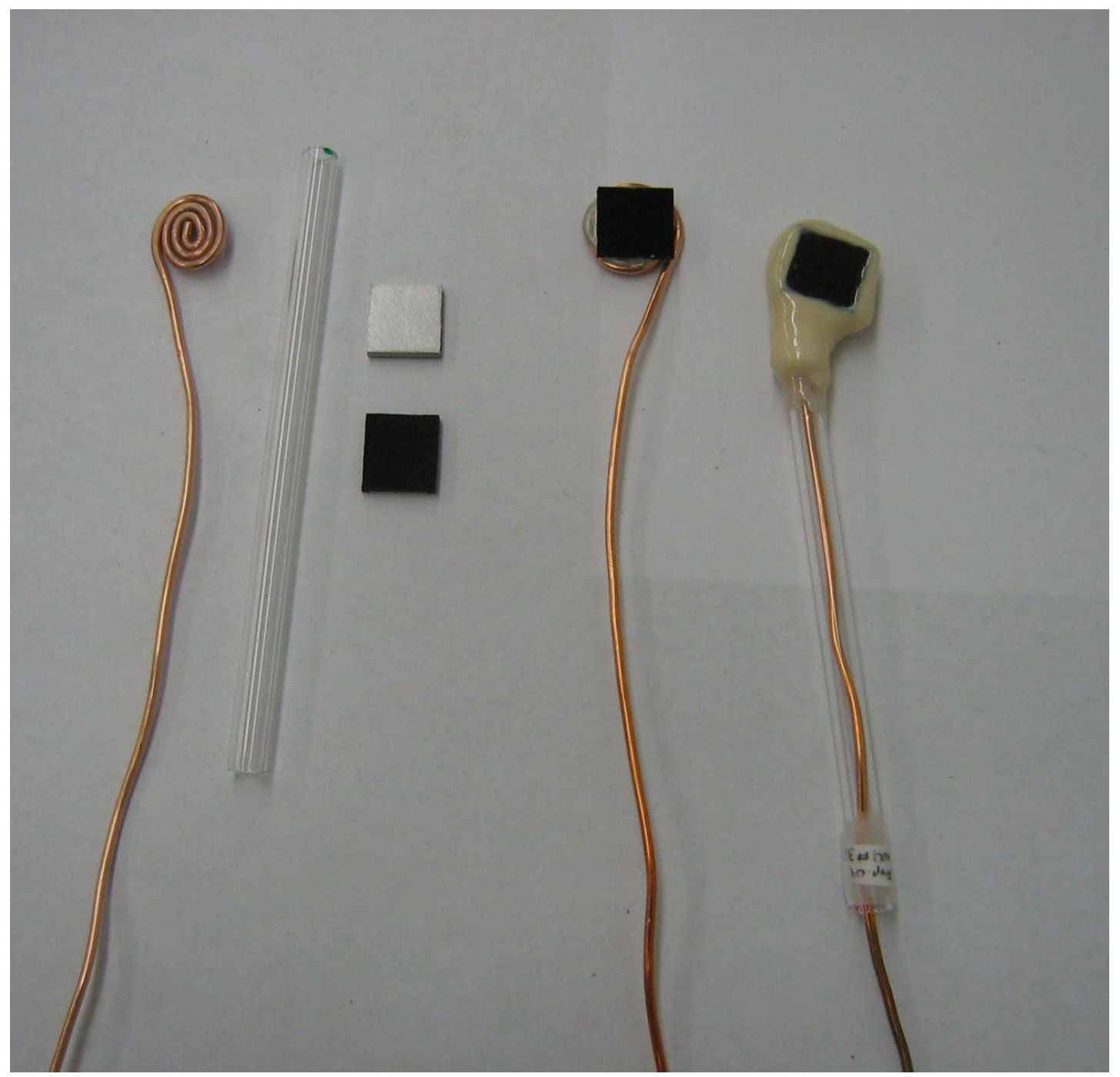


FIGURE 10 - Typical electrode (right) as well as components to make the electrode (left) and partially assembled (middle).

Finally, the exposed surface area of the electrode needed to be calculated. Using a copying machine, under $200 \%$ magnification with printing colors inverted, negatives of the faces of each electrode were copied and printed. Next, under 200\% magnification, a piece of grid paper from a laboratory notebook was also copied and printed. A $1 \mathrm{~cm}^{2}$ section was cut from a piece of grid paper and the exposed face of the copied sample electrode was cut out. Each of these were then weighed individually and since the density of each piece of paper is the same, a simple ratio can be made in order to estimate the area of the exposed sample electrode face shown in Equation (11).

$$
\frac{\text { mass paper sample }}{\text { mass of } 1 \mathrm{~cm}^{2}} \times 1 \mathrm{~cm}^{2}=\text { area of electrode face }
$$

\section{Photoelectrochemical Characterization}

Current Density-Voltage (J-V): J-V testing is performed by applying a voltage over a range, at a specific rate of scanning, and reading the subsequent current density exhibited by the electrode. This can be performed in both two and three electrode configurations in order to discern different characteristics. A scanning rate of $25 \mathrm{mV} / \mathrm{s}$ was used in this experiment. Solar-to-Hydrogen efficiency can be attained from J-V testing in a two electrode set-up while the inherent energy properties and kinetics of the sample can be known by performing three-electrode $\mathrm{J}-\mathrm{V}$ measurements. The net photoactivity of the 
sample is measured by performing $\mathrm{J}-\mathrm{V}$ testing both with and without illumination and subtracting the subsequent photoactivity with illumination from the photoactivity without illumination. As a PEC testing rule, n-type materials should be tested in the positive bias direction and p-type materials should be tested in the negative bias direction. This is because scanning in the opposite direction can inflict harm to the semiconductor due to a massive amount of holes congregating at the semiconductor surface, causing it to exhibit conductivity similar to a metal and in turn cause corrosion at the surface. The potential at which the current exhibited under no illumination onsets marks the inversion onset within the semiconductor. The onset of photocurrent in the illuminated $\mathrm{J}-\mathrm{V}$ scans indicate the potential at which charge carriers are able to overcome the energy requirement to drive the redox reaction as shown in figure 6 .

Open Circuit Potential (OCP): As discussed in the theory section, the bands within a semiconductor will flatten out upon illumination. Bending occurs in opposite direction for n-type and p-type semiconductors. Measuring the potential of a semiconductor in an open circuit before and after illumination will therefore reveal its conductivity type. This test is called OCP. A positive potential shift upon illumination indicates p-type conductivity. OCP measurements were performed using a three-electrode setup in $1 \mathrm{M}$ $\mathrm{KOH}$ solution with AM $1.5(100 \mathrm{~mW} / \mathrm{cm} 2)$ illumination. Acidic solution proved to be corrosive for the samples. In order for the potential readings to reach equilibrium, OCP was typically performed for a duration of about 180 seconds, with light being turned on at 90 seconds. 


\section{RESULTS AND DISCUSSION OF RESULTS}

\section{Synthesis and Characterization of HP Plasma Oxidized Hematite NW Arrays}

Hematite Nanowire Arrays were successfully synthesized using the Atmospheric Plasma Jet Reactor. Imaging done using SEM show an array of NWs that were approximately 5-10 micrometers in length, with bases approximately $100-300 \mathrm{~nm}$ in diameter that tapered off to about 5-10 nm at the tips. in Figure 11 that the NWs were $100-300 \mathrm{~nm}$ at the base and tapered down to approximately $10 \mathrm{~nm}$ at the tips, and up to 510 microns in length.

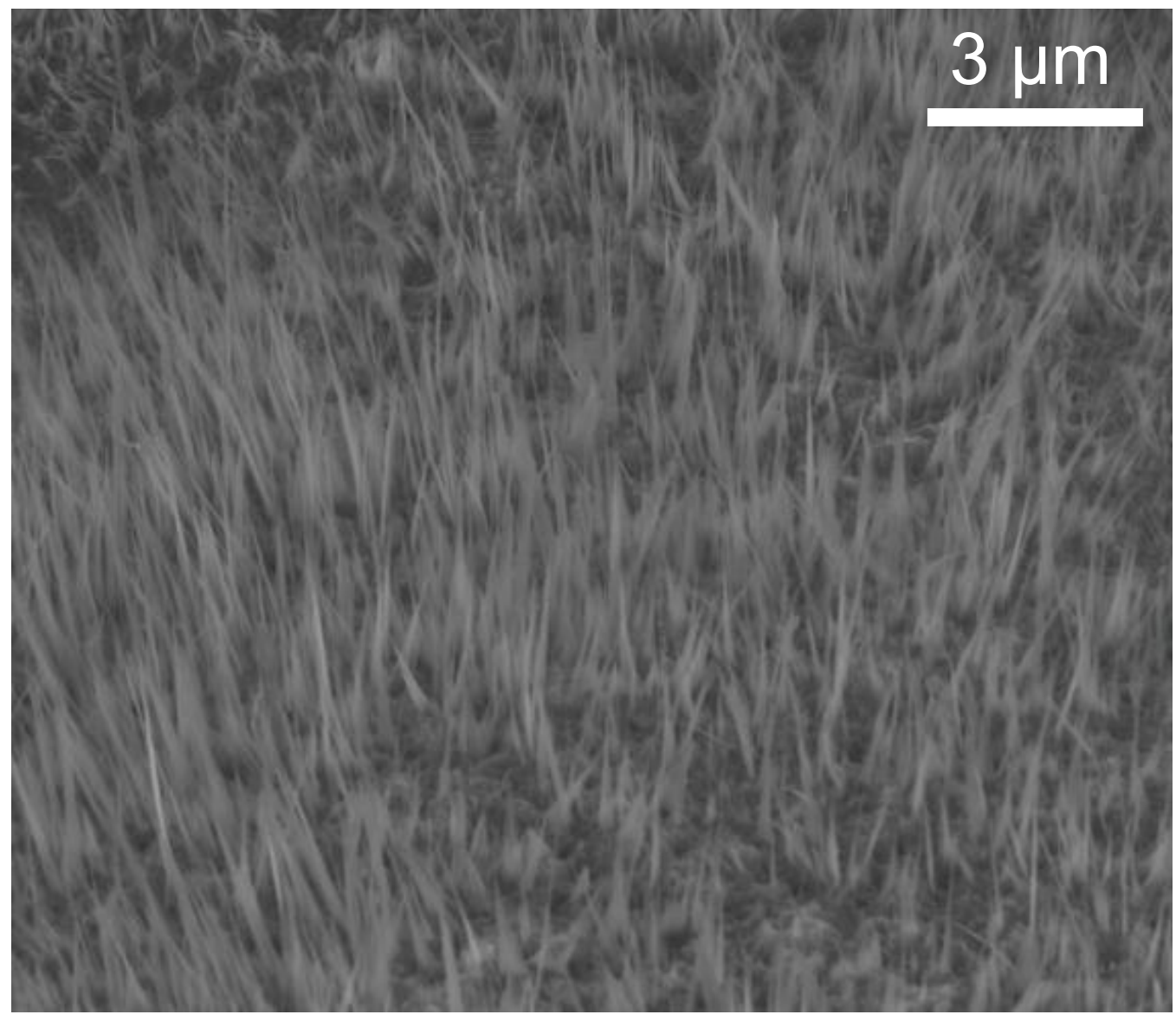


FIGURE 11 - SEM of HP Plasma Hematite NW Array

In an attempt to characterize the semiconductor composition, the semiconductor was subjected Raman spectroscopy testing. The result was compared against a bulk hematite powder as well as Raman spectras for hematite from other publications ${ }^{94,96,123}$ and corresponds well with the $\alpha-\mathrm{Fe}_{2} \mathrm{O}_{3}$ phase.

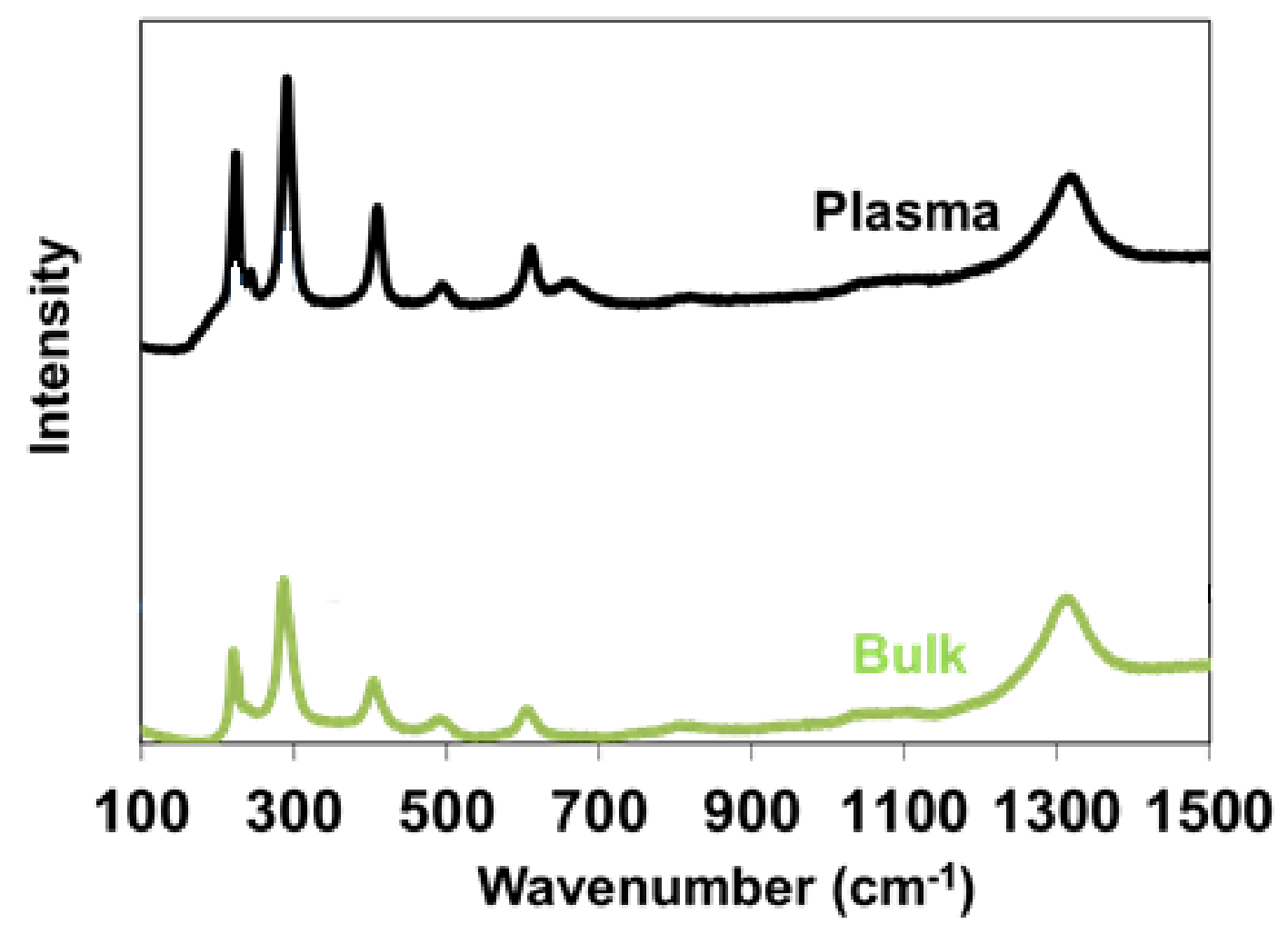

FIGURE 12 - Raman Spectra comparing HP Plasma oxidized hematite NW Arrays against a known bulk hematite powder.

In order to determine the band gap of the atmospheric plasma synthesized hematite nanowire arrays, UV-Vis spectroscopy was performed using diffuse reflectance (DR) 
measurements due to the opaqueness of the samples. Absorbance data was obtained by converting the diffuse reflectance data using equation (12).

$$
A=R_{\max }-R
$$

Where $R_{\max }$ signifies the highest $\mathrm{R}$ value after the dip in DR. With Equation 12, the maximum point of absorbance is set to zero at the point just before the dip signifying the band gap. The band gap can then be attained using an extrapolation procedure explained in detail in the references ${ }^{124}$. An absorbance plot for the Atmospheric Plasma synthesized hematite nanowire arrays is shown in figure 13. The linear portion of the curve was extrapolated indicating a $2.1 \mathrm{eV}$ band gap that agrees with estimates from literature $^{125}$.

The $2.1 \mathrm{eV}$ band gap in hematite is indirect and has been explained in literature to be due to the $\mathrm{Fe}^{3+} 3 \mathrm{~d}$ to $3 \mathrm{~d}$ excitation ${ }^{70}$. 


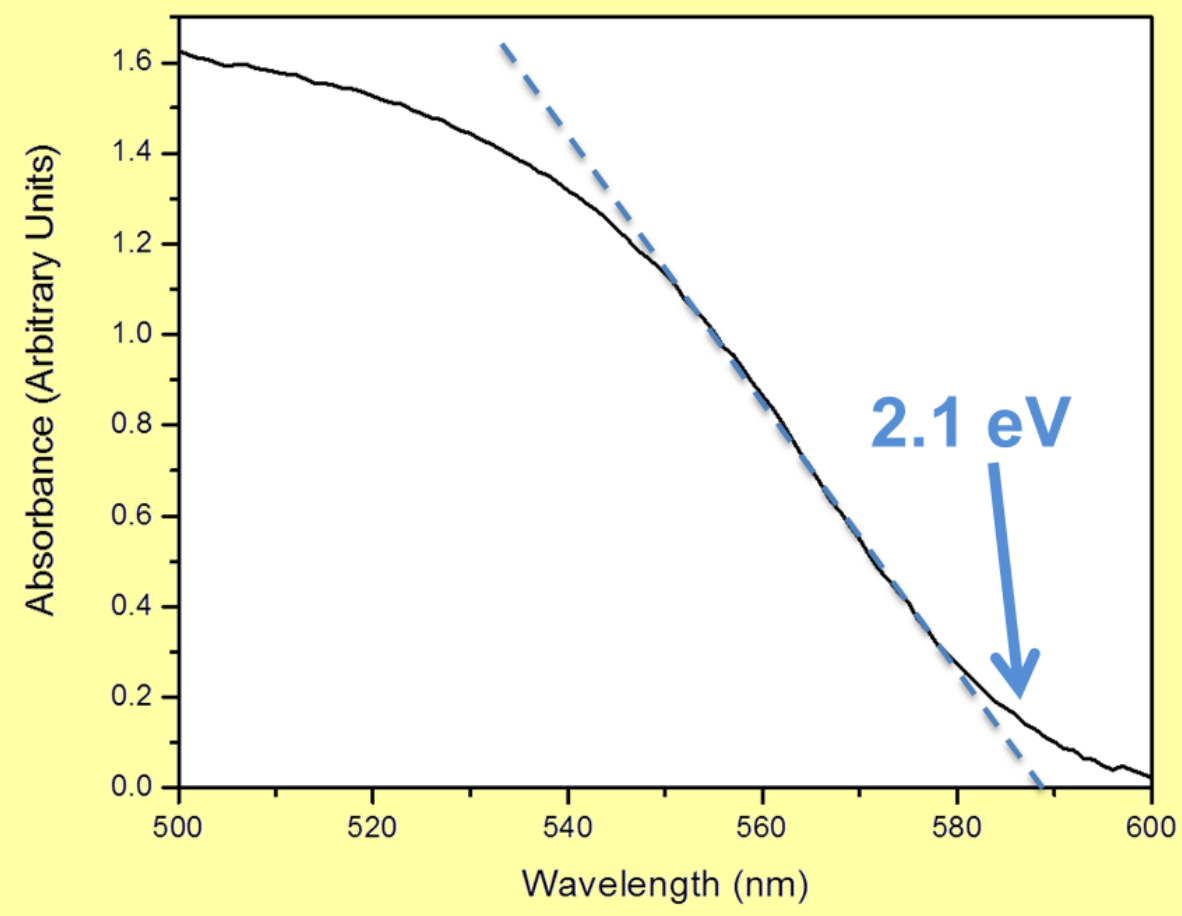

FIGURE 13 - UV-Vis Spectrum of HP Plasma Hematite Nanowire Array

The conductivity type, meaning $\mathrm{n}$ - or p-type, of the semiconductor was determined by carrying out Open circuit potential (OCP) measurements. The test is done in a three electrode set-up using $\mathrm{Ag} / \mathrm{AgCl}$ as the reference electrode in a $1 \mathrm{M} \mathrm{KOH}$ electrolytic bath. The illumination source used provides AM 1.5 light. Measurements begin by measuring potential over time under no illumination, then suddenly illuminating the sample after a prespecified amount of time has passed (between 60 and 90 seconds). Upon illumination, there will be a resultant change in potential. The direction of this shift indicates n-type behavior for a negative shift in potential and p-type for a positive shift in potential. The resultant shift in the synthesized hematite nanowire arrays indicates n-type behavior and is shown in figure 14 . 


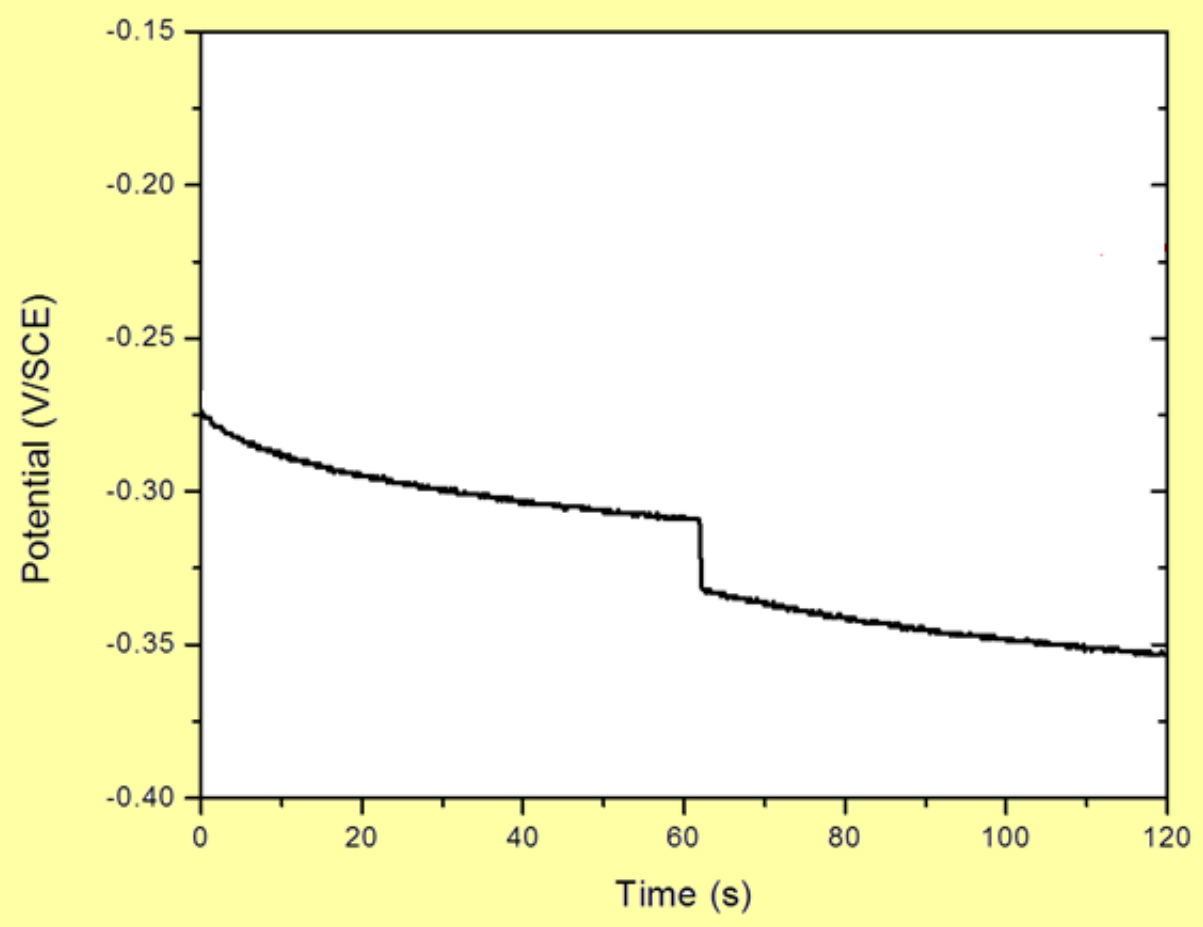

FIGURE 14 - OCP measurement of Hematite NW Array electrode indicating n-type conductivity.

\section{HP Plasma versus LP Plasma Synthesis}

At first, hematite nanowire arrays synthesized by atmospheric plasma showed little to no photoactivity. To understand why these samples exhibited no photoactivity while those synthesized under vacuum did, compared both samples under TEM in order to discern and differences between the two samples. Atmospheric and Low pressure plasma samples showed nearly identical structural qualities however, arrays synthesized under ambient conditions exhibited a 1 to $5 \mathrm{~nm}$ amorphous oxide sheath at the surface of the nanowires as shown in the HRTEM image in figure 15a. At first, there was confusion 
as to where this amorphous layer came from. One thought was that it was as a result of synthesis; the other was that it was a result of PEC testing, or perhaps both! In an attempt to remove the oxide layer from the surface of the nanowire array, the samples were submerged in dilute hydrofluoric acid for several seconds. Statistical HRTEM imaging of many nanowires from the samples before and after acid etching indicates that the surface amorphous oxide layer can be completely removed via acid etching, exposing the single crystal oxide surface.

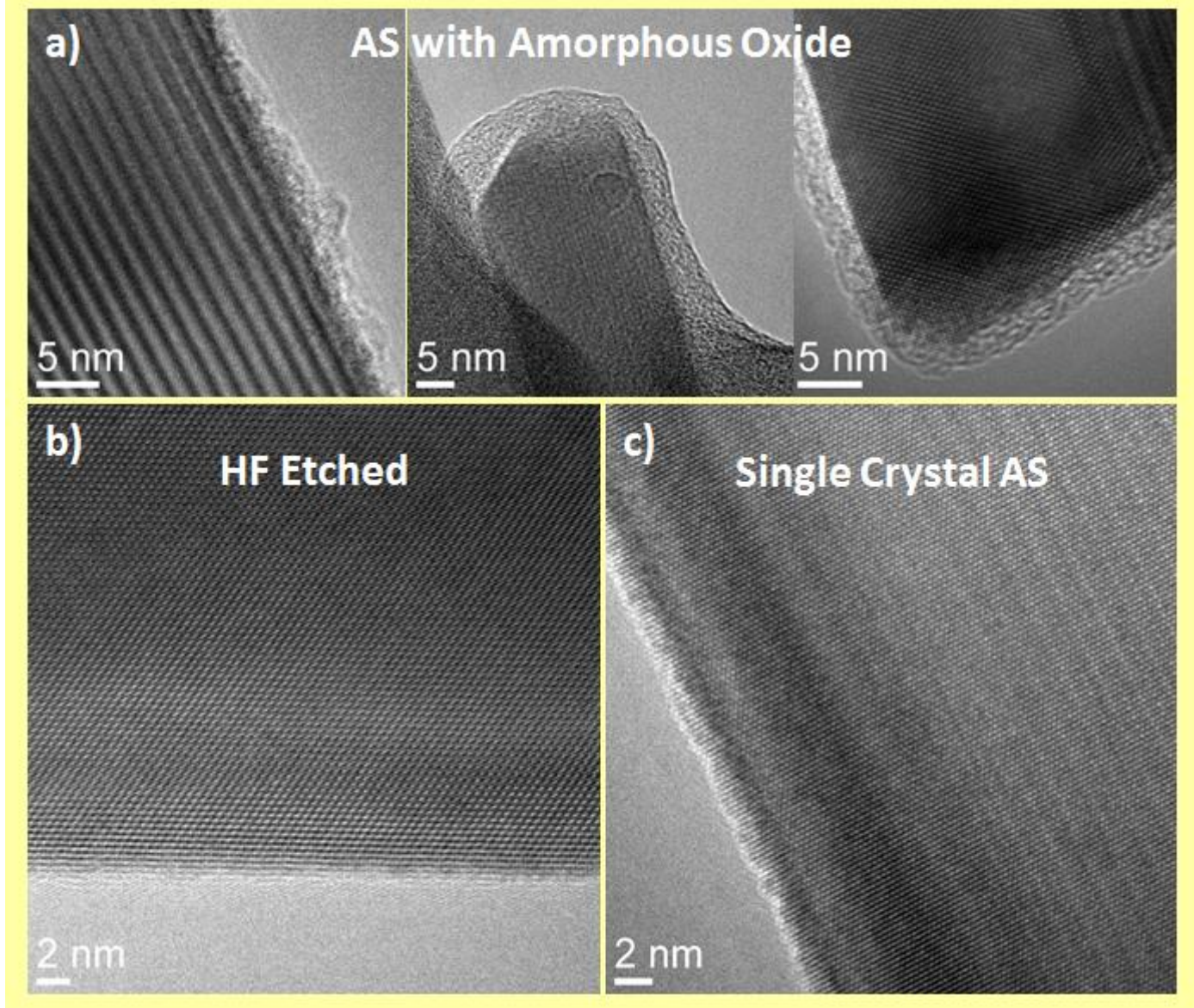

FIGURE 15 - HRTEM imaging of hematite nanowires synthesized by HP Plasma

Oxidation showing (a) the amorphous oxide layer created during synthesis, (b) a hematite 
nanowire after HF etching showing a removed amorphous oxide layer and (c) a hematite nanowire showing no amorphous oxide after improvements in synthesis conditions.

Once the amorphous oxide sheath had been removed from the hematite nanowires, J-V characteristics were performed again and the acid etched sample showed a significant photoactivity when it had shown none with the oxide layer. Figure 16 shows the J-V characteristic curves for both before and after acid etched hematite nanowire arrays synthesized by atmospheric plasma. One observation besides the rise in photoconductivity is the negative shift of the photocurrent onset towards more negative values after acid treatment.

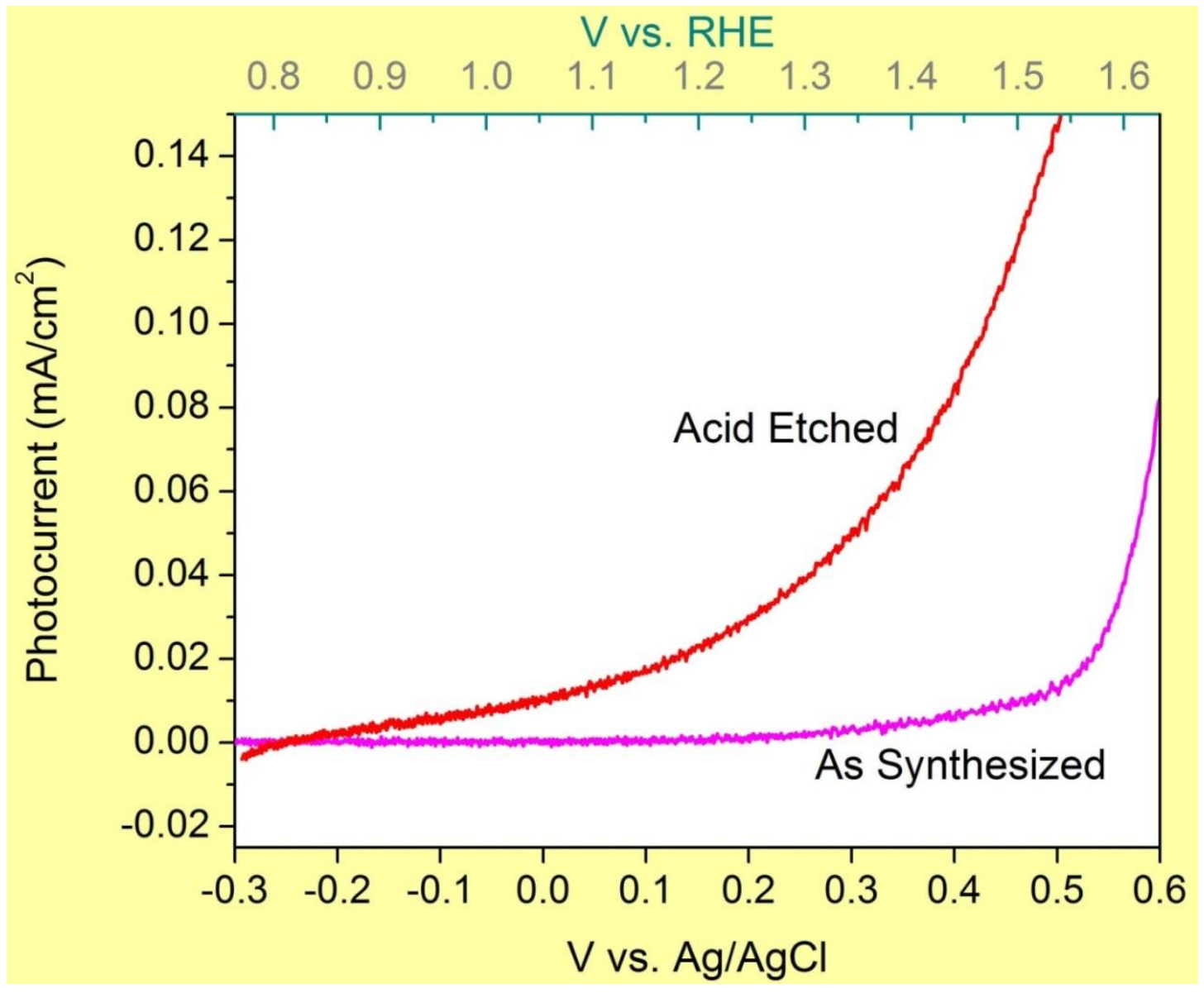


FIGURE 16 - Light minus Dark IV Characteristics graph showing the increase in photoactivity of a hematite nanowire array with the amorphous oxide layer present compared to the same electrode after HF Etching.

Over time, synthesis conditions improved enabling the synthesis of hematite nanowire arrays using atmospheric plasma that exhibited no amorphous oxide layer. As shown in figure $15 \mathrm{c}$, these nanowires show single crystalline nature extending all the way to the surface of the nanowire. These samples showed similar characteristics to that of the previously synthesized HP plasma samples after acid etching including the negative shift of the photocurrent onset. By introducing catalytic treatments to the nanowire array, a more negative shift in onset potentials would be expected ${ }^{41}$. With the new synthesis conditions, As-synthesized HP Plasma hematite NW array electrodes that exhibit no amorphous oxide surface layer can be reproducibly to have a significant photoactivity, although with some variance inherent to the synthesis procedure, as can be seen from figure 17. Further engineering of the process in order to control nanowire length, diameter and density would fix this variance in photoactivity. 


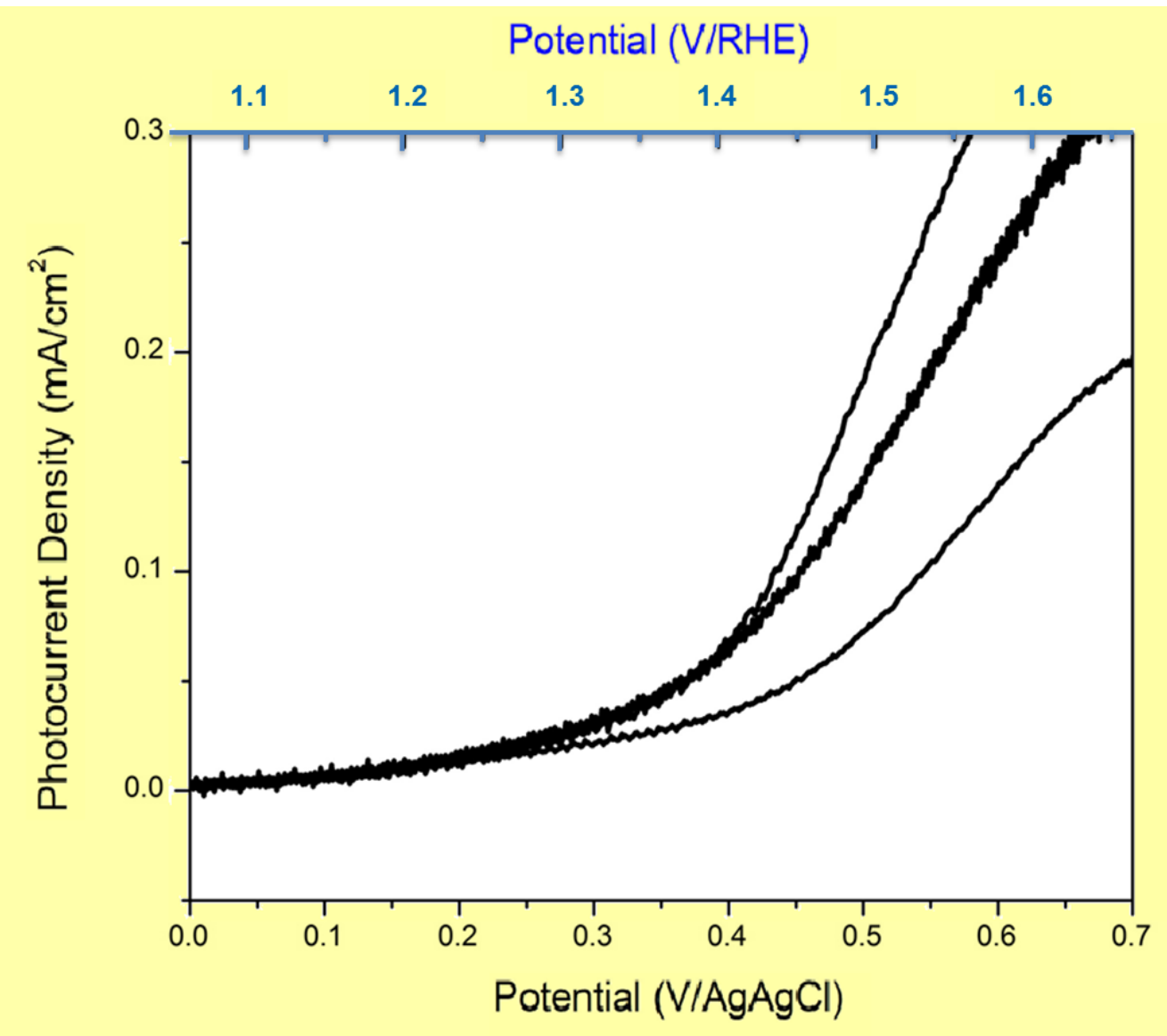

FIGURE 17 - Light minus dark IV characteristics graph for several HP Plasma synthesized hematite nanowire array samples showing a significant photoactivity.

The finding that crystallinity at the NW surface dramatically effects photoactivity of the semiconductor device is a major finding that may have promise in improving other nano-morphologies of metal oxides. 


\section{Confirmation of Photoactivity due to Nanowires}

In order to verify that photoactivity produced by the sample was indeed as a result of the nanowires present on the sample, and not simply the thin film interfacial layer present in plasma synthesized samples, NWs were removed from the sample delicately, (in order to leave the interfacial layer intact) and the sample was subsequently ran through $\mathrm{J}-\mathrm{V}$ characteristics. The resultant $\mathrm{J}-\mathrm{V}$ curve showed that after removal of the NWs from the electrode surface, there was a significant drop in photoactivity (figure 18) which implies that photoactivity of hematite nanowire arrays is indeed due to the nanowires present on the sample. 


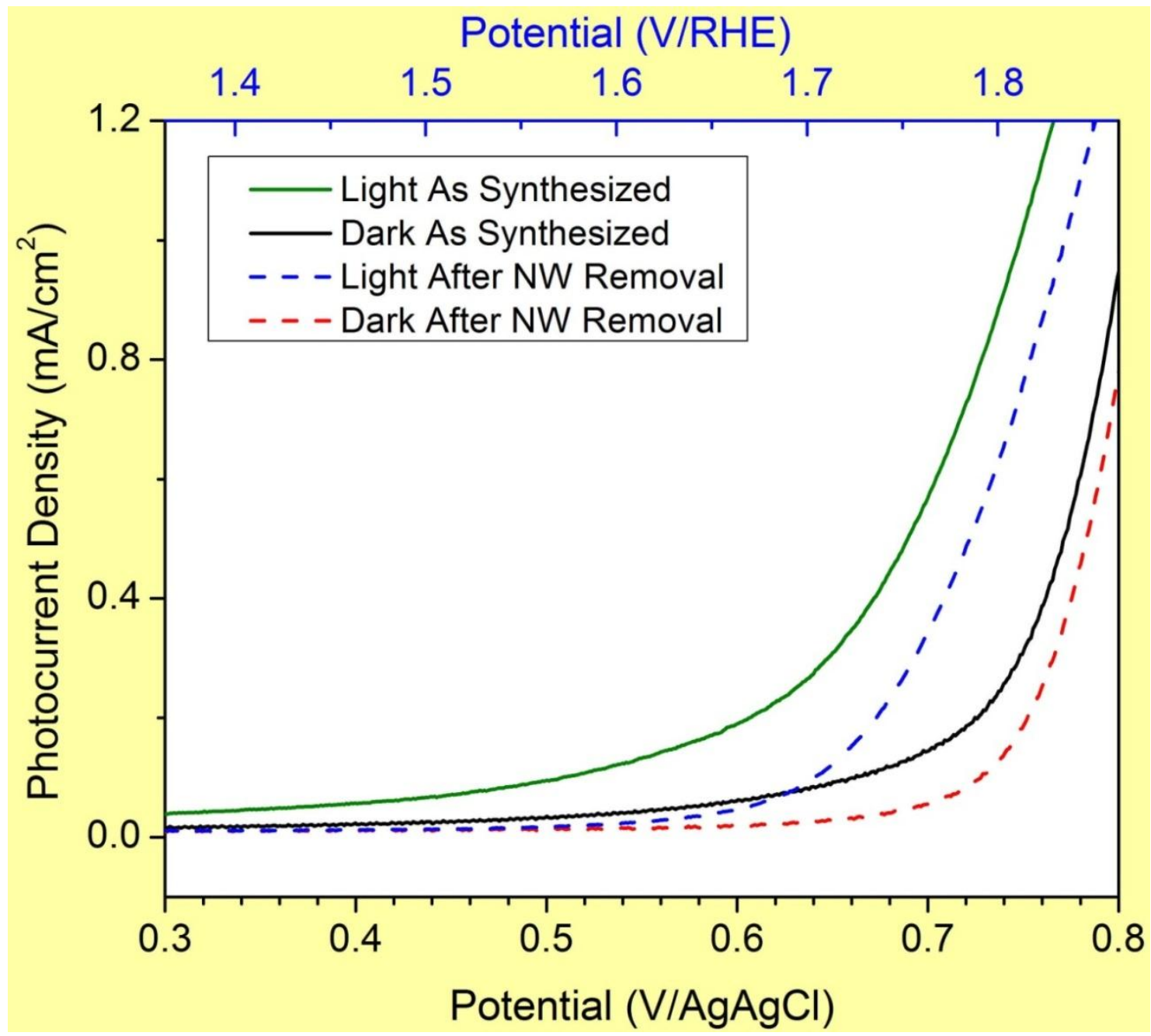

FIGURE 18 - Light and Dark IV Characteristics graph hematite nanowire arrays before and after nanowire removal.

\section{Light Intensity Dependence}

In order to determine whether the hematite NW arrays synthesized by HP plasma were capable of making use of concentrated light, a light intensity dependence test was performed. By scanning at a constant voltage (.3V) at many different light intensities, a sample's resultant photoactivity can help one determine how the incoming light intensity affects the photoactivity. As shown in Figure 19, the photoactivity of hematite nanowire 
arrays synthesized by HP Plasma has a linear dependence on light intensity. This shows that transport in the nanowire array structure does not limit the amount of photoactivity that can be exhibited. This implies that as the concentration of light on the electrode increase, the photoactivity will increase by the same factor. Thus by magnifying light on the electrode less of the electrode would be needed to produce the same photoactivity. If the semiconductor was diffusion limited, at higher light intensities, there would not be as much of a gain in photoactivity and would increase in more of a square-root like nature.

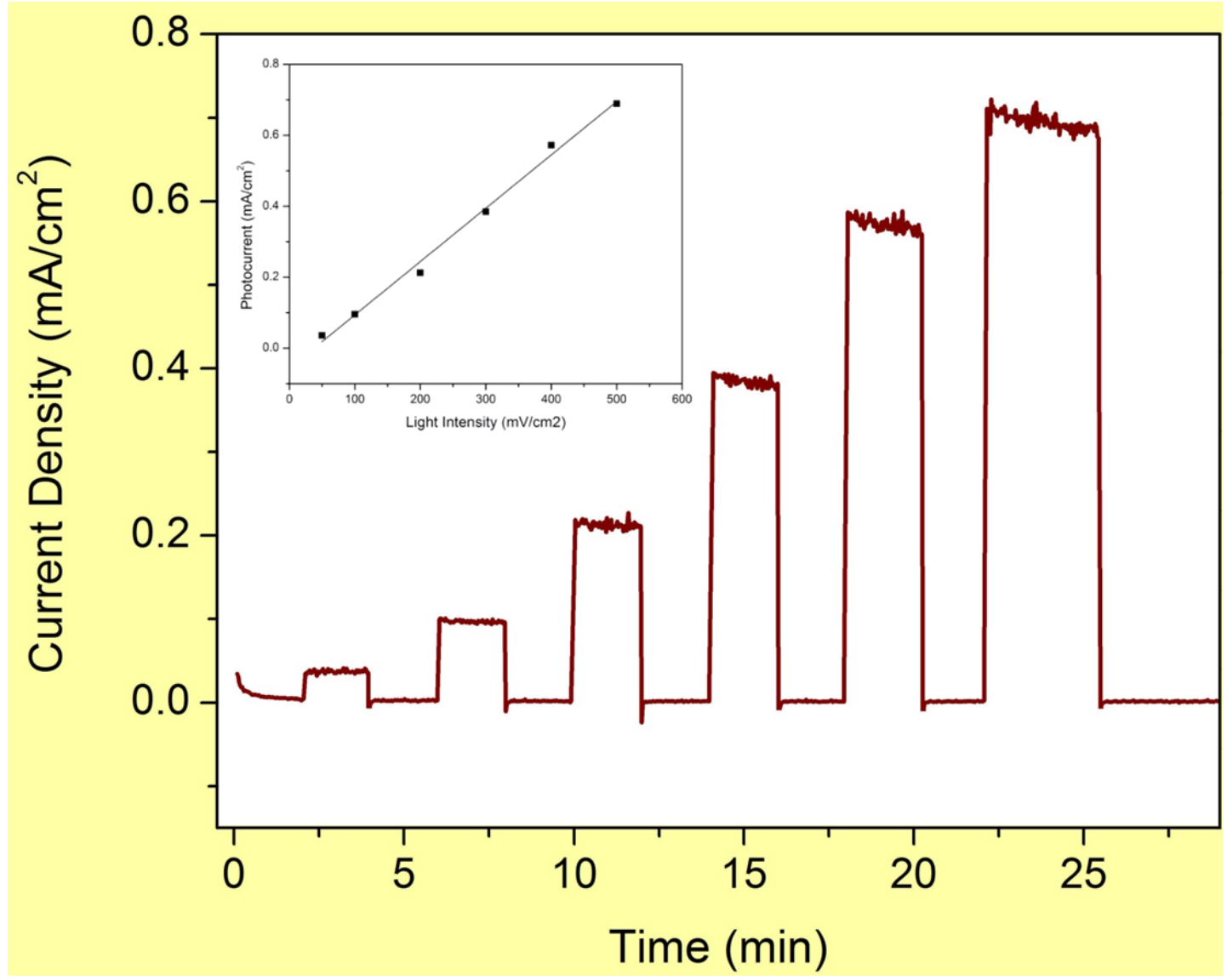


FIGURE 19 - IV Characteristics Graph at constant voltage under different illumination intensities with inset showing that the photoactivity of hematite nanowire arrays exhibit linear dependence on light intensity.

TEM studies of NW arrays after PEC testing (Figure 20) show that the NWs retain the original structure as well as the oxygen vacancy super-lattice.

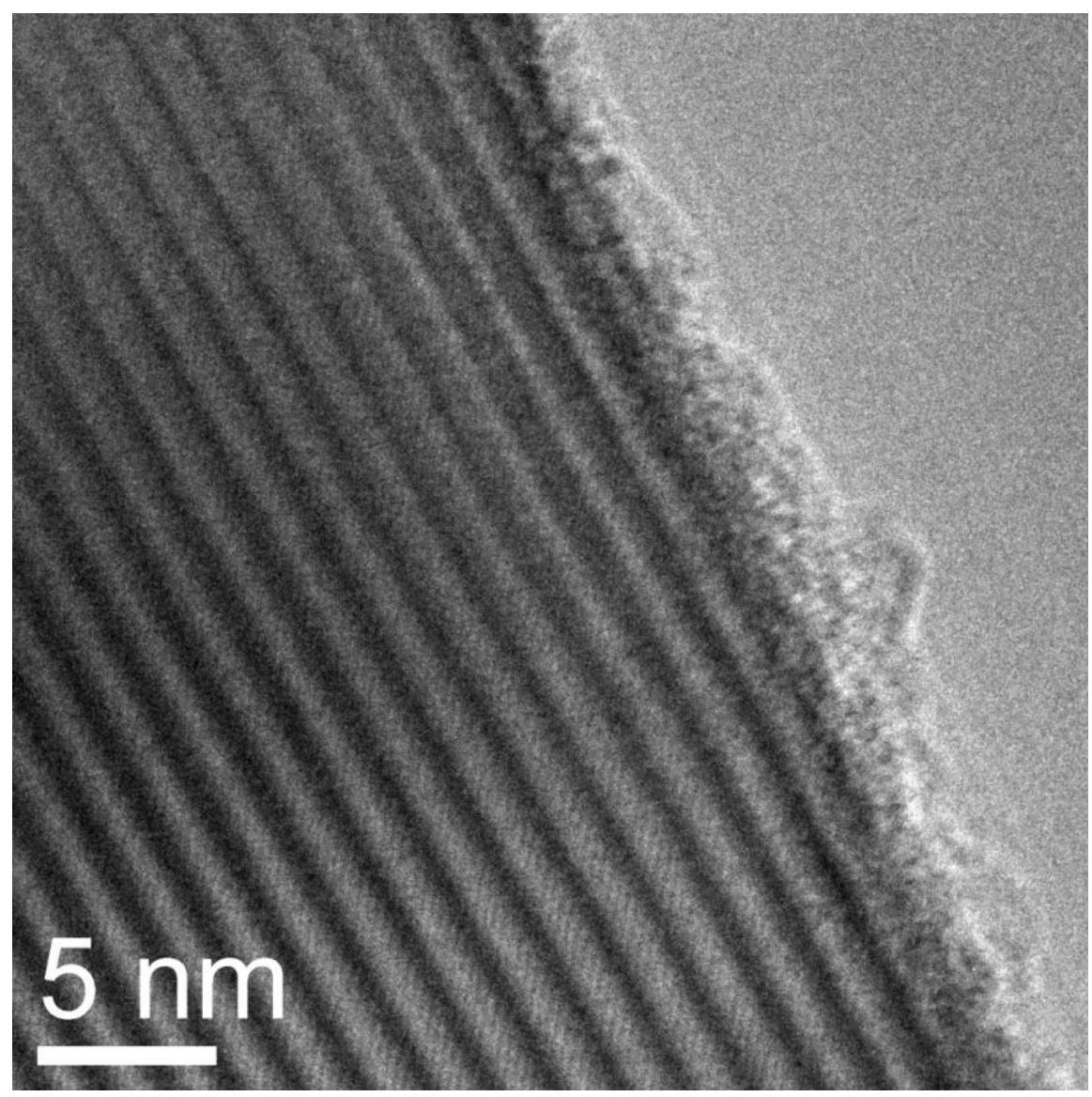

FIGURE 20 - HRTEM imaging of HP plasma sample after PEC testing showing oxygen vacancies as well as amorphous oxide sheath 


\section{CONCLUSIONS}

- Hematite NW Arrays produced by HP Plasma are nearly identical to those synthesized by low pressure plasma except that under some synthesis conditions, an amorphous oxide sheath is present on the nanowire surface, inhibiting photoactivity.

- If an amorphous oxide sheath is present, it can be removed via acid etching and photoactivity can be dramatically increased.

- By performing IV characteristics on an as synthesized hematite nanowire array, then removing nanowires from the array and performing IV characteristics again, a decreased photoactivity was observed and the photoactivity produced by the electrode was shown to be due to the nanowires and not the thin interfacial layer present.

- The photoactivity of hematite nanowire arrays shows a linear dependence with light intensity. Indicating transport within the array does not limit photoconductivity, allowing concentrated light to be used to increase photoactivity. 


\section{RECOMMENDATIONS}

- Long-term characterization needs to be performed to address degradation.

- Optimization of growth conditions will take place to vary NW density and size to address issues of light absorbance and photoactivity.

- Doping with silicon and labeling with quantum dots or Au nanosphere will be attempted to increase conductivity and absorbance respectively.

- P-type doping of hematite NW array will be attempted.

- Transfer of the hematite NW array to a transparent conductive oxide will be attempted in order to take advantage of tandem cell configurations. 


\section{APPENDIX A}

Instructions for fabricating an electrode for use in photoelectrochemical studies

1) MIX Loctite 9464 Hysol Epoxy until white. Make sure to replace cap on epoxy!!!!

2) Preheat Blue M Oven to $160 \mathrm{C}$ (this is approximately $120 \mathrm{C}$ ).

3) Remove any oxide from back of your sample via sandpaper or filing.

4) Take 14AWG wire and needle nose pliers and twist wire in form of a lollipop with the head being approximately the same size as your sample.

(c)

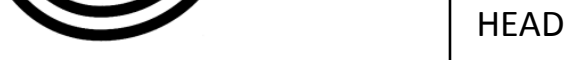

5) Sand off the head of your copper wire to remove any oxide.

6) Mix Circuit Works Silver Conductive Epoxy A \& B (only has 10 minute work time so use fast!)

7) Apply a layer of Silver Epoxy on both the head of the wire and on back contact of sample

8) Place Electrode in oven until silver epoxy is cured (should feel hard $\approx 30$ mins)

9) Use a file to cut a section from one of the long tubes in the friedrich and dimmock box down to to a 3-4 inch section.

10) When Silver epoxy has cured, sheath the long part of the electrode with the glass tube

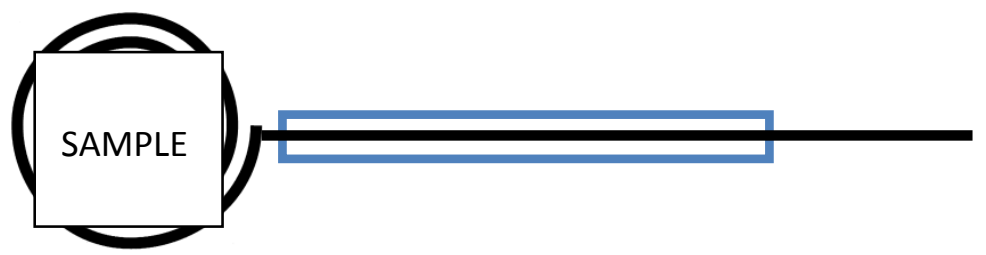

11) Apply Hysol 9462 to all sides of your electrode except top. Make sure all bubbles in epoxy are covered up.

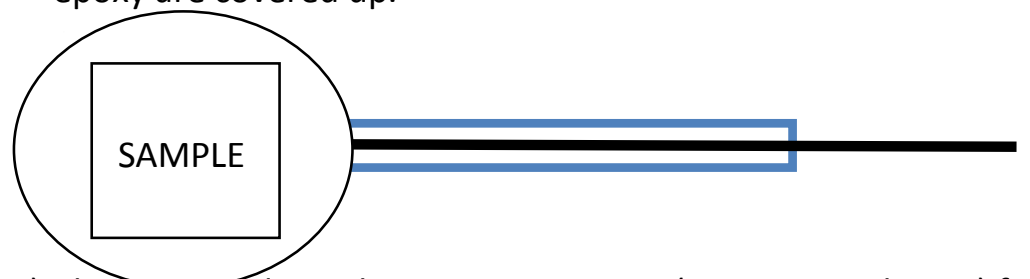

12) Place Etectrode in Blue M Oven at $110 \mathrm{C}$ (approximately 80C) for 1 hour

13) MIX Hysol E120HP Epoxy (Allow 1 to 1.5 hours before using)

14) Apply a thin layer of E120HP Epoxy on top of all places the 9462 Epoxy was applied (9462 is an excellent mechanical bonding epoxy while E120HP has excellent chemical resistance)

15) Place Sample back in oven for 2 hours OR leave in ambient conditions overnight (place sample in sample holder parallel to the ground) 


\section{REFERENCES}

(1) Fujishima, A.; Honda, K.: Electrochemical Photolysis of Water at a Semiconductor Electrode. Nature 1972, 238, 37-+.

(2) Emery, K.; Myers, D.: Reference Solar Spectral Irradiance: Air Mass 1.5. Center, R. E. R. D., Ed.; National Renewable Energy Laboratory, 2009; Vol. 2009.

(3) Nozik, A. J.; Memming, R.: Physical chemistry of semiconductor-liquid interfaces. Journal of Physical Chemistry 1996, 100, 13061-13078.

(4) Bott, A. W.: Electrochemistry of Semiconductors. Current Separations $1998,17$.

(5) Gerischer, H. Z.: Physcal Chemistry (Munich) 1960, 26, 223.

(6) Nozik, A. J.: Photoelectrochemistry - Applications to solar-energy conversion. Annual Review of Physical Chemistry 1978, 29, 189-222.

(7) Gerischer, H. Z.: Physcal Chemistry (Munich) 1961, 27, 48.

(8) Bak, T.; Nowotny, J.; Rekas, M.; Sorrell, C. C.: Photo-electrochemical hydrogen generation from water using solar energy. Materials-related aspects. International Journal of Hydrogen Energy 2002, 27, 991-1022.

(9) Gerischer, H.: Solar photoelectrolysis with semiconductor electrodes. In Topics in Applied Physics; Springer, 1979; Vol. 31; pp 115-172.

(10) Khaselev, O.; Turner, J. A.: A monolithic photovoltaicphotoelectrochemical device for hydrogen production via water splitting. Science 1998, 280, 425-427.

(11) Varghese, O. K.; Grimes, C. A.: Appropriate strategies for determining the photoconversion efficiency of water photo electrolysis cells: A review with examples using titania nanotube array photoanodes. Solar Energy Materials and Solar Cells 2008, 92, 374-384.

(12) Kim, D. H.; Anderson, M. A.: Photoelectrocatalytic degradation of formic-acid using a porous $\mathrm{TiO} 2$ thin-film electrode. Environmental Science \& Technology 1994, 28, 479-483.

(13) Ogden, A. D.; Gole, J. L.; Fedorov, A. G.: Optical and electronic properties of semiconducting nanostructures for photocatalytic hydrogen generation. Journal of Nanoelectronics and Optoelectronics 2007, 2, 269-277.

(14) Mathur, S.; Sivakov, V.; Shen, H.; Barth, S.; Cavelius, C.; Nilsson, A.; Kuhn, P.: Nanostructured films of iron, tin and titanium oxides by chemical vapor deposition. Elsevier Science Sa, 2006; pp 88-93.

(15) Hardee, K. L.; Bard, A. J.: Semiconductor electrodes .1. chemical vapordeposition and application of polycrystalline n-type titanium dioxide electrodes to photosensitized electrolysis of water. J. Electrochem. Soc. 1975, 122, 739-742.

(16) van de Krol, R.; Liang, Y. Q.; Schoonman, J.: Solar hydrogen production with nanostructured metal oxides. Journal of Materials Chemistry 2008, 18, 2311-2320.

(17) Jitputti, J.; Suzuki, Y.; Yoshikawa, S.: Synthesis of TiO2 nanowires and their photocatalytic activity for hydrogen evolution. Catalysis Communications 2008, 9, 1265-1271. 
(18) Park, J. H.; Kim, S.; Bard, A. J.: Novel carbon-doped TiO2 nanotube arrays with high aspect ratios for efficient solar water splitting. Nano Letters 2006, 6, 2428.

(19) Khan, S. U. M.; Sultana, T.: Photoresponse of n-TiO2 thin film and nanowire electrodes. Solar Energy Materials and Solar Cells 2003, 76, 211-221.

(20) Rensmo, H.; Lindstrom, H.; Sodergren, S.; Willstedt, A. K.; Solbrand, A.; Hagfeldt, A.; Lindquist, S. E.: Photocurrent losses in Nanocrystalline/Nanoporous TiO2 electrodes due to electrochemically active species in the electrolyte. J. Electrochem. Soc. 1996, 143, 3173-3178.

(21) Nowotny, J.; Bak, T.; Nowotny, M. K.; Sheppard, L. R.: Titanium dioxide for solar-hydrogen I. Functional properties. 2007; pp 2609-2629.

(22) Gratzel, M.: Photoelectrochemical cells. Nature 2001, 414, 338-344.

(23) Su, L. Y.; Zhang, L. G.; Fang, J. H.; Xu, M. H.; Lu, Z. H.: Electrochromic and photoelectrochemical behavior of electrodeposited tungsten trioxide films. Solar Energy Materials and Solar Cells 1999, 58, 133-140.

(24) Gondal, M. A.; Hameed, A.; Yamani, Z. H.: Laser induced photocatalytic splitting of water over WO3 catalyst. Energy Sources 2005, 27, 1151-1165.

(25) Marsen, B.; Miller, E. L.; Paluselli, D.; Rocheleau, R. E.: Progress in sputtered tungsten trioxide for photoelectrode applications. Pergamon-Elsevier Science Ltd, 2007; pp 3110-3115.

(26) Baeck, S. H.; Jaramillo, T.; Stucky, G. D.; McFarland, E. W.: Controlled electrodeposition of nanoparticulate tungsten oxide. Nano Letters 2002, 2, 831-834.

(27) Hotchandani, S.; Kamat, P. V.: Photoelectrochemistry of semiconductor ZNO particulate films. J. Electrochem. Soc. 1992, 139, 1630-1634.

(28) Ahn, K. S.; Shet, S.; Deutsch, T.; Jiang, C. S.; Yan, Y. F.; Al-Jassim, M.; Turner, J.: Enhancement of photoelectrochemical response by aligned nanorods in $\mathrm{ZnO}$ thin films. Journal of Power Sources 2008, 176, 387-392.

(29) Jaramillo, T. F.; Baeck, S. H.; Kleiman-Shwarsctein, A.; Choi, K. S.; Stucky, G. D.; McFarland, E. W.: Automated electrochemical synthesis and photoelectrochemical characterization of $\mathrm{Zn1-xCoxO}$ thin films for solar hydrogen production. Journal of Combinatorial Chemistry 2005, 7, 264-271.

(30) Mavroides, J. G.; Kafalas, J. A.; Kolesar, D. F.: Photoelectrolysis of water in cells with SRTiO3 anodes. Applied Physics Letters 1976, 28, 241-243.

(31) Wrighton, M. S.; Ellis, A. B.; Wolczanski, P. T.; Morse, D. L.; Abrahamson, H. B.; Ginley, D. S.: Strontium-titanate photoelectrodes - efficient photoassisted electrolysis of water at zero applied potential. J. Am. Chem. Soc. 1976, 98, 2774-2779.

(32) Watanabe, T.; Fujishima, A.; Honda, K.: Photoelectrochemical reactions at SrTiO3 single-crystal electrode. Bulletin of the Chemical Society of Japan 1976, 49, 355-358.

(33) El Zayat, M. Y.; Saed, A. O.; El-Dessouki, M. S.: Photoelectrochemical properties of dye sensitized $\mathrm{Zr}$-doped SrTiO3 electrodes. International Journal of Hydrogen Energy 1998, 23, 259-266.

(34) Glasscock, J. A.; Barnes, P. R. F.; Plumb, I. C.; Savvides, N.: Enhancement of photoelectrochemical hydrogen production from hematite thin films by the introduction of Ti and Si. Journal of Physical Chemistry C 2007, 111, 16477-16488. 
(35) Hu, Y. S.; Kleiman-Shwarsctein, A.; Stucky, G. D.; McFarland, E. W.: Improved photoelectrochemical performance of Ti-doped alpha-Fe2O3 thin films by surface modification with fluoride. Chemical Communications 2009, 2652-2654.

(36) Hu, Y. S.; Kleiman-Shwarsctein, A.; Forman, A. J.; Hazen, D.; Park, J. N.; McFarland, E. W.: Pt-doped alpha-Fe2O3 thin films active for photoelectrochemical water splitting. Chemistry of Materials 2008, 20, 3803-3805.

(37) Ingler, W. B.; Khan, S. U. M.: Photoresponse of spray pyrolytically synthesized magnesium-doped iron(III) oxide (p-Fe2O3) thin films under solar simulated light illumination. Thin Solid Films 2004, 461, 301-308.

(38) Ingler, W. B.; Khan, S. U. M.: Photoresponse of spray pyrolytically synthesized copper-doped $\mathrm{p}-\mathrm{Fe} 2 \mathrm{O} 3$ thin film electrodes in water splitting. International Journal of Hydrogen Energy 2005, 30, 821-827.

(39) Ingler, W. B.; Khan, S. U. M.: A self-driven p/n-Fe2O3 tandem photoelectrochemical cell for water splitting. Electrochemical and Solid State Letters 2006, 9, G144-G146.

(40) Iordanova, N.; Dupuis, M.; Rosso, K. M.: Charge transport in metal oxides: A theoretical study of hematite alpha-Fe2O3. Journal of Chemical Physics 2005, $122,10$.

(41) Kay, A.; Cesar, I.; Gratzel, M.: New benchmark for water photooxidation by nanostructured alpha-Fe2O3 films. J. Am. Chem. Soc. 2006, 128, 15714-15721.

(42) Kennedy, J. H.; Frese, K. W.: Photooxidation of water at alpha-Fe2O3 electrodes. J. Electrochem. Soc. 1977, 124, C130-C130.

(43) Kennedy, J. H.; Frese, K. W.: Flatband potentials and donor densities of polycrystalline alpha-Fe2O3 determined from Mott-Schottky plots. J. Electrochem. Soc. 1978, 125, 723-726.

(44) Kennedy, J. H.; Anderman, M.; Shinar, R.: Photoactivity of polycrystalline alpha-Fe2O3 electrodes doped with group IV-A elements. J. Electrochem. Soc. 1981, 128, 2371-2373.

(45) Kennedy, J. H.; Anderman, M.: Photoelectrolysis of water at alpha-Fe2O3 electrodes in acidic solution. J. Electrochem. Soc. 1983, 130, 848-852.

(46) Khader, M. M.; Vurens, G. H.; Kim, I. K.; Salmeron, M.; Somorjai, G. A.: Photoassisted catalytic dissociation of $\mathrm{H} 2 \mathrm{O}$ to produce hydrogen on partially reduced alpha-Fe2O3. J. Am. Chem. Soc. 1987, 109, 3581-3585.

(47) Khan, S. U. M.; Akikusa, J.: Photoelectrochemical splitting of water at nanocrystalline n-Fe2O3 thin-film electrodes. Journal of Physical Chemistry B 1999, $103,7184-7189$.

(48) Kleiman-Shwarsctein, A.; Hu, Y. S.; Forman, A. J.; Stucky, G. D.; McFarland, E. W.: Electrodeposition of alpha-Fe2O3 doped with Mo or $\mathrm{Cr}$ as photoanodes for photocatalytic water splitting. Journal of Physical Chemistry C 2008, $112,15900-15907$.

(49) Kumari, S.; Tripathi, C.; Singh, A. P.; Chauhan, D.; Shrivastav, R.; Dass, S.; Satsangi, V. R.: Characterization of Zn-doped hematite thin films for photoelectrochemical splitting of water. Current Science 2006, 91, 1062-1064.

(50) Kumari, S.; Singh, A. P.; Tripathi, C.; Chauhan, D.; Dass, S.; Shrivastav, R.; Gupta, V.; Sreenivas, K.; Satsangi, V. R.: Enhanced photoelectrochemical response of Zn-dotted hematite. International Journal of Photoenergy 2007, 6. 
(51) Lindgren, T.; Wang, H. L.; Beermann, N.; Vayssieres, L.; Hagfeldt, A.; Lindquist, S. E.: Aqueous photoelectrochemistry of hematite nanorod array. Solar Energy Materials and Solar Cells 2002, 71, 231-243.

(52) Quinn, R. K.; Nasby, R. D.; Baughman, R. J.: Photoassisted electrolysis of water using single-crystal alpha-Fe2O3. Materials Research Bulletin 1976, 11, 10111017.

(53) Sartoretti, C. J.; Ulmann, M.; Alexander, B. D.; Augustynski, J.; Weidenkaff, A.: Photoelectrochemical oxidation of water at transparent ferric oxide film electrodes. Chemical Physics Letters 2003, 376, 194-200.

(54) Shinar, R.; Kennedy, J. H.: Photoactivity of doped alpha Fe-2O3 electrodes. Solar Energy Materials 1982, 6, 323-335.

(55) Leygraf, C.; Hendewerk, M.; Somorjai, G. A.: Mg-doped and Si-doped iron-oxides for the photocatalyzed production of hydrogen from water by visible-light (2.2 eV-less-than-or-equal-to-H-nu-less-than-or-equal-to-2.7-eV). Journal of Catalysis 1982, 78, 341-351.

(56) Dareedwards, M. P.; Goodenough, J. B.; Hamnett, A.; Trevellick, P. R.: Electrochemistry and photoelectrochemistry of Iron (III) Oxide. Journal of the Chemical Society-Faraday Transactions I 1983, 79, 2027-2041.

(57) Itoh, K.; Bockris, J. O.: Thin-film photoelectrochemistry - iron - oxide. J. Electrochem. Soc. 1984, 131, 1266-1271.

(58) Turner, J. E.; Hendewerk, M.; Parmeter, J.; Neiman, D.; Somorjai, G. A.: The characterization of doped iron-oxide electrodes for the photodissociation of water stability, optical, and electronic properties. J. Electrochem. Soc. 1984, 131, 1777-1783.

(59) Leland, J. K.; Bard, A. J.: Photoelectrochemistry of colloidal semiconducting iron-oxide polymorphs. Journal of Physical Chemistry 1987, 91, 50765083.

(60) Bernasik, A.; Janowski, J.; Hirschwald, W.; Stolze, F.; Nowotny, J.: Effect of surface-treatment on segregation of impurities in hematite.. Journal of Materials Science 1991, 26, 2527-2532.

(61) Uekawa, N.; Watanabe, M.; Kaneko, K.; Mizukami, F.: Mixed-valence formation in highly oriented T-doped iron-oxide film. Journal of the Chemical SocietyFaraday Transactions 1995, 91, 2161-2166.

(62) Cherepy, N. J.; Liston, D. B.; Lovejoy, J. A.; Deng, H. M.; Zhang, J. Z.: Ultrafast studies of photoexcited electron dynamics in gamma- and alpha-Fe2O3 semiconductor nanoparticles. Journal of Physical Chemistry B 1998, 102, 770-776.

(63) Rosso, K. M.; Smith, D. M. A.; Dupuis, M.: An ab initio model of electron transport in hematite (alpha-Fe2O3) basal planes. Journal of Chemical Physics 2003, $118,6455-6466$.

(64) Watanabe, A.; Kozuka, H.: Photoanodic properties of sol-gel-derived $\mathrm{Fe} 2 \mathrm{O} 3$ thin films containing dispersed gold and silver particles. Journal of Physical Chemistry B 2003, 107, 12713-12720.

(65) Gondal, M. A.; Hameed, A.; Yamani, Z. H.; Suwaiyan, A.: Production of hydrogen and oxygen by water splitting using laser induced photo-catalysis over Fe2O3. Applied Catalysis a-General 2004, 268, 159-167. 
(66) Aroutiounian, V. M.; Arakelyan, V. M.; Shahnazaryan, G. E.; Stepanyan, G. M.; Khachaturyan, E. A.; Turner, J. A.: Investigations of the structure of the iron oxide semiconductor-electrolyte interface. 2006; pp 325-331.

(67) Benjelloun, D.; Bonnet, J. P.; Doumerc, J. P.; Launay, J. C.; Onillon, M.; Hagenmuller, P.: Anisotropy of the electrical-properties of iron-oxide alph-Fe2O3. Materials Chemistry and Physics 1984, 10, 503-518.

(68) Bjorksten, U.; Moser, J.; Gratzel, M.: Photoelectrochemical studies on nanocrystalline hematite films. Chemistry of Materials 1994, 6, 858-863.

(69) Cesar, I.; Kay, A.; Martinez, J. A. G.; Gratzel, M.: Translucent thin film Fe2O3 photoanodes for efficient water splitting by sunlight: Nanostructure-directing effect of Si-doping. J. Am. Chem. Soc. 2006, 128, 4582-4583.

(70) Duret, A.; Gratzel, M.: Visible light-induced water oxidation on mesoscopic alpha-Fe2O3 films made by ultrasonic spray pyrolysis. Journal of Physical Chemistry B 2005, 109, 17184-17191.

(71) Arai, T.; Konishi, Y.; Iwasaki, Y.; Sugihara, H.; Sayama, K.: Highthroughput screening using porous photoelectrode for the development of visible-lightresponsive semiconductors. Journal of Combinatorial Chemistry 2007, 9, 574-581.

(72) Ahmed, S. M.; Leduc, J.; Haller, S. F.: Photoelectrochemical and impedance characteristics of specular hematite .1. photoelectrochemical, parallel conductance and trap rate studies. Journal of Physical Chemistry 1988, 92, 6655-6660.

(73) Sanchez, C.; Sieber, K. D.; Somorjai, G. A.: the photoelectrochemistry of niobium doped alpha-Fe2(O3). Journal of Electroanalytical Chemistry 1988, 252, 269290.

(74) Leygraf, C.; Hendewerk, M.; Somorjai, G.: The preparation and selected properties of $\mathrm{Mg}$-doped para-type iron-oxide as a photo-cathode for the photoelectrolysis of water using visible-light. Journal of Solid State Chemistry 1983, 48, 357-367.

(75) Ingler, W. B.; Baltrus, J. P.; Khan, S. U. M.: Photoresponse of p-type zincdoped iron(III) oxide thin films. J. Am. Chem. Soc. 2004, 126, 10238-10239.

(76) Fan, Z. Y.; Wen, X. G.; Yang, S. H.; Lu, J. G.: Controlled p- and n-type doping of Fe2O3 nanobelt field effect transistors. Applied Physics Letters 2005, 87, 3.

(77) Aroutiounian, V. M.; Arakelyan, V. M.; Shahnazaryan, G. E.; Stepanyan, G. M.; Khachaturyan, E. A.; Wang, H.; Turner, J. A.: Photoelectrochemistry of semiconductor electrodes made of solid solutions in the system Fe2O3-Nb2O5. Solar Energy 2006, 80, 1098-1111.

(78) Aroutiounian, V. M.; Arakelyan, V. M.; Shahnazaryan, G. E.; Stepanyan, G. M.; Turner, J. A.; Khaselev, O.: Investigation of ceramic Fe2O3 < Ta > photoelectrodes for solar energy photoelectrochemical converters. International Journal of Hydrogen Energy 2002, 27, 33-38.

(79) Sartoretti, C. J.; Alexander, B. D.; Solarska, R.; Rutkowska, W. A.; Augustynski, J.; Cerny, R.: Photoelectrochemical oxidation of water at transparent ferric oxide film electrodes. Journal of Physical Chemistry B 2005, 109, 13685-13692.

(80) Zhong, L. S.; Hu, J. S.; Liang, H. P.; Cao, A. M.; Song, W. G.; Wan, L. J.: Self-assembled 3D flowerlike iron oxide nanostructures and their application in water treatment. Advanced Materials 2006, 18, 2426-+. 
(81) Cui, H. T.; Zayat, M.; Levy, D.: Sol-gel synthesis of nanoscaled spinels using propylene oxide as a gelation agent. Journal of Sol-Gel Science and Technology 2005, 35, 175-181.

(82) Gash, A. E.; Tillotson, T. M.; Satcher, J. H.; Poco, J. F.; Hrubesh, L. W.; Simpson, R. L.: Use of epoxides in the sol-gel synthesis of porous iron(III) oxide monoliths from $\mathrm{Fe}(\mathrm{III})$ salts. Chemistry of Materials 2001, 13, 999-1007.

(83) Wang, H. Z.; Zhang, X. T.; Liu, B.; Zhao, H. L.; Li, Y. C.; Huang, Y. B.; $\mathrm{Du}, \mathrm{Z}$. L.: Synthesis and characterization of single crystal alpha-Fe2O3 nanobelts. Chemistry Letters 2005, 34, 184-185.

(84) Woo, K.; Lee, H. J.; Ahn, J. P.; Park, Y. S.: Sol-gel mediated synthesis of Fe2O3 nanorods. Advanced Materials 2003, 15, 1761-+.

(85) Wu, D. H.; Zhang, Z. X.; Xu, L. F.; Shen, L. L.; Wang, X.: Preparation of monodispersed nano alpha-Fe2O3 single crystal particles by refluxing hydrothermal method. Chinese Journal of Inorganic Chemistry 2004, 20, 31-36.

(86) Iwasaki, M.; Hara, M.; Ito, S.: Synthesis of nanometer-sized hematite single crystals through NAC-FAS method. Journal of Materials Science 2000, 35, 943949.

(87) Nagahama, J.; Yumoto, H.: Application of Fe oxide films prepared by PVD methods to protect Fe metal from corrosion. Elsevier Science Sa, 2003; pp 658-661.

(88) Beermann, N.; Vayssieres, L.; Lindquist, S. E.; Hagfeldt, A.:

Photoelectrochemical studies of oriented nanorod thin films of hematite. J. Electrochem. Soc. 2000, 147, 2456-2461.

(89) Vayssieres, L.; Guo, J. H.; Nordgren, J.: Aqueous chemical growth of alpha-Fe2O3-alpha-Cr2O3 nanocomposite thin films. Journal of Nanoscience and Nanotechnology 2001, 1, 385-388.

(90) Hardee, K. L.; Bard, A. J.: Semiconductor electrodes .5. application of chemically vapor-deposited iron-oxide films to photosensitized electrolysis. $J$. Electrochem. Soc. 1976, 123, 1024-1026.

(91) Chueh, Y. L.; Lai, M. W.; Liang, J. Q.; Chou, L. J.; Wang, Z. L.: Systematic study of the growth of aligned arrays of alpha-Fe2O3 and $\mathrm{Fe} 3 \mathrm{O} 4$ nanowires by a vapor-solid process. Adv. Funct. Mater. 2006, 16, 2243-2251.

(92) Wang, R. M.; Chen, Y. F.; Fu, Y. Y.; Zhang, H.; Kisielowski, C.: Bicrystalline hematite nanowires. Journal of Physical Chemistry B 2005, 109, 12245 12249.

(93) Han, Q.; Xu, Y. Y.; Fu, Y. Y.; Zhang, H.; Wang, R. M.; Wang, T. M.; Chen, Z. Y.: Defects and growing mechanisms of alpha-Fe2O3 nanowires. Chemical Physics Letters 2006, 431, 100-103.

(94) Fu, Y. Y.; Wang, R. M.; Xu, J.; Chen, J.; Yan, Y.; Narlikar, A.; Zhang, H.: Synthesis of large arrays of aligned alpha-Fe2O3 nanowires. Chemical Physics Letters 2003, 379, 373-379.

(95) Chen, Z.; Cvelbar, U.; Mozetic, M.; He, J.; Sunkara, M. K.: Long Range Ordering of Oxygen Vacancy Planes in alpha-Fe2O3 Nanowires and Nanobelts. Chemistry of Materials 2008, Submitted.

(96) Cvelbar, U.; Chen, Z. Q.; Sunkara, M. K.; Mozetic, M.: Spontaneous Growth of Superstructure alpha-Fe2O3 Nanowire and Nanobelt Arrays in Reactive Oxygen Plasma. Small 2008, 4, 1610-1614. 
(97) Cvelbar, U.; Ostrikov, K.; Levchenko, I.; Mozetic, M.; Sunkara, M. K.: Control of morphology and nucleation density of iron oxide nanostructures by electric conditions on iron surfaces exposed to reactive oxygen plasmas. Applied Physics Letters 2009, 94.

(98) Schrebler, R.; Bello, K.; Vera, F.; Cury, P.; Munoz, E.; del Rio, R.; Meier, H. G.; Cordova, R.; Dalchiele, E. A.: An electrochemical deposition route for obtaining alpha-Fe2O3 thin films. Electrochemical and Solid State Letters 2006, 9, C110-C113.

(99) Zotti, G.; Schiavon, G.; Zecchin, S.; Casellato, U.: Electrodeposition of amorphous $\mathrm{Fe} 2 \mathrm{O} 3$ films by reduction of iron perchlorate in acetonitrile. J. Electrochem. Soc. 1998, 145, 385-389.

(100) Schrebler, R.; Llewelyn, C.; Vera, F.; Cury, P.; Munoz, E.; del Rio, R.; Meier, H. G.; Cordova, R.; Dalchiele, E. A.: An electrochemical deposition route for obtaining alpha-Fe2O3 thin films - II. EQCM study and semiconductor properties. Electrochemical and Solid State Letters 2007, 10, D95-D99.

(101) !!! INVALID CITATION !!!

(102) Kocha, S. S.; Peterson, M. W.; Arent, D. J.; Redwing, J. M.; Tischler, M. A.; Turner, J. A.: Electrochemical investigation of the gallium nitride-aqueous electrolyte interface. J. Electrochem. Soc. 1995, 142, L238-L240.

(103) Akikusa, J.; Khan, S. U. M.: Photoelectrolysis of water to hydrogen in p$\mathrm{SiC} / \mathrm{Pt}$ and $\mathrm{p}-\mathrm{SiC} / \mathrm{n}-\mathrm{TiO} 2$ cells. International Journal of Hydrogen Energy 2002, 27, 863870.

(104) Damilano, B.; Grandjean, N.; Vezian, S.; Massies, J.: InGaN heterostructures grown by molecular beam epitaxy: from growth mechanism to optical properties. Elsevier Science Bv, 2001; pp 466-470.

(105) Ho, I. H.; Stringfellow, G. B.: Solid phase immiscibility in GaInN. Applied Physics Letters 1996, 69, 2701-2703.

(106) Orton, J. W.; Foxon, C. T.: Group III nitride semiconductors for short wavelength light-emitting devices. Reports on Progress in Physics 1998, 61, 1-+.

(107) Shioda, T.; Sugiyama, M.; Shimogaki, Y.; Nakano, Y.: Selective area metal-organic vapor-phase epitaxy of $\mathrm{InN}, \mathrm{GaN}$ and InGaN covering whole composition range. Elsevier Science Bv, 2009; pp 2809-2812.

(108) Deutsch, T. G.; Head, J. L.; Turner, J. A.: Photoelectrochemical characterization and durability analysis of GaInPN epilayers. J. Electrochem. Soc. 2008, 155, B903-B907.

(109) Deutsch, T. G.; Koval, C. A.; Turner, J. A.: III-V nitride epilayers for photoelectrochemical water splitting: GaPN and GaAsPN. Journal of Physical Chemistry B 2006, 110, 25297-25307.

(110) Khaselev, O.; Turner, J. A.: Electrochemical stability of p-GaInP2 in aqueous electrolytes toward photoelectrochemical water splitting. J. Electrochem. Soc. 1998, 145, 3335-3339.

(111) Khaselev, O.; Turner, J. A.: Anodic oxidation of GaInP2. Corrosion Science 2000, 42, 1831-1838.

(112) Khaselev, O.; Bansal, A.; Turner, J. A.: High-efficiency integrated multijunction photovoltaic/electrolysis systems for hydrogen production. PergamonElsevier Science Ltd, 2001; pp 127-132. 
(113) Aroutiounian, V. M.; Arakelyan, V. M.; Shahnazaryan, G. E.: Metal oxide photoelectrodes for hydrogen generation using solar radiation-driven water splitting. Solar Energy 2005, 78, 581-592.

(114) Rocheleau, R. E.; Miller, E. L.: Photoelectrochemical production of hydrogen: Engineering loss analysis. International Journal of Hydrogen Energy 1997, $22,771-782$.

(115) Bolton, J. R.: Solar photoproduction of hydrogen: A review. Solar Energy 1996, 57, 37-50.

(116) Vayssieres, L.; Sathe, C.; Butorin, S. M.; Shuh, D. K.; Nordgren, J.; Guo, J. H.: One-dimensional quantum-confinement effect in alpha-Fe2O3 ultrafine nanorod arrays. Advanced Materials 2005, 17, 2320-+.

(117) Wen, X. G.; Wang, S. H.; Ding, Y.; Wang, Z. L.; Yang, S. H.: Controlled growth of large-area, uniform, vertically aligned arrays of alpha-Fe2O3 nanobelts and nanowires. Journal of Physical Chemistry B 2005, 109, 215-220.

(118) Kim, C. H.; Chun, H. J.; Kim, D. S.; Kim, S. Y.; Park, J.; Moon, J. Y.; Lee, G.; Yoon, J.; Jo, Y.; Jung, M. H.; Jung, S. I.; Lee, C. J.: Magnetic anisotropy of vertically aligned alpha-Fe2O3 nanowire array. Applied Physics Letters 2006, 89, 3.

(119) Yu, T.; Zhu, Y. W.; Xu, X. J.; Yeong, K. S.; Shen, Z. X.; Chen, P.; Lim, C. T.; Thong, J. T. L.; Sow, C. H.: Substrate-friendly synthesis of metal oxide nanostructures using a hotplate. Small 2006, 2, 80-84.

(120) Vayssieres, L.; Beermann, N.; Lindquist, S. E.; Hagfeldt, A.: Controlled aqueous chemical growth of oriented three-dimensional crystalline nanorod arrays: Application to iron(III) oxides. Chemistry of Materials 2001, 13, 233-235.

(121) Mozetic, M.; Cvelbar, U.; Sunkara, M. K.; Vaddiraju, S.: A method for the rapid synthesis of large quantities of metal oxide nanowires at low temperatures. Advanced Materials 2005, 17, 2138-+.

(122) Kumar, V.; Kim, J. H.; Pendyala, C.; Chernomordik, B.; Sunkara, M. K.: Gas-Phase, Bulk Production of Metal Oxide Nanowires and Nanoparticles Using a Microwave Plasma Jet Reactor. Journal of Physical Chemistry C 2008, 112, 1775017754.

(123) de Faria, D. L. A.; Venâncio Silva, S.; de Oliveira, M. T.: Raman microspectroscopy of some iron oxides and oxyhydroxides. Journal of Raman Spectroscopy 1997, 28, 873-878.

(124) Murphy, A. B.: Band-gap determination from diffuse reflectance measurements of semiconductor films, and application to photoelectrochemical watersplitting. Solar Energy Materials and Solar Cells 2007, 91, 1326-1337.

(125) Kennedy, J. H.; Frese, K. W.: Photo-oxidation of water at alpha-Fe2O3 electrodes. J. Electrochem. Soc. 1978, 125, 709-714. 


\section{VITA}

Harry Benjamin Russell achieved his bachelor's degree in Chemical Engineering at the University of Louisville in 2009. He will continue to pursue a doctoral degree in Chemical Engineering from the University of Louisville, following his MEng work. 